\title{
A REVIEW OF HYBRID INTEGRAL TRANSFORM SOLUTIONS IN FLUID FLOW PROBLEMS WITH HEAT OR MASS TRANSFER AND UNDER NAVIER-STOKES EQUATIONS FORMULATIONS
}

\author{
Renato M. Cotta ${ }^{1,2 *}$ Kleber M. Lisboa ${ }^{2}$, Marcos F. Curi $^{1,3}$, Stavroula Balabani $^{4}$, \\ João N.N. Quaresma ${ }^{4,5}$, Jesus S. Perez-Guerrero ${ }^{6}$, Emanuel N. Macedo ${ }^{5}$, Nelson S. Amorim ${ }^{5}$ \\ ${ }^{1}$ Mechanical Engineering Department, POLI \& COPPE, Universidade Federal do Rio de Janeiro \\ Cx. Postal 68503, Cidade Universitária, Rio de Janeiro, RJ, Brazil, 21945-970 \\ ${ }^{2}$ Directorate General of Nuclear and Technological Development - DGDNTM, Brazilian Navy, Rio \\ de Janeiro, RJ, Brazil \\ ${ }^{3}$ Centro Federal de Educação Tecnológica Celso Suckow da Fonseca, CEFET/RJ, Itaguaí, RJ, \\ Brazil \\ ${ }^{4}$ Department Mechanical Engineering Dept., University College London, London, UK, WC1E 7JE \\ ${ }^{5}$ Graduate Program in Natural Resource Engineering in the Amazon \\ Institute of Technology, Universidade Federal do Pará, Belém, PA, Brazil \\ ${ }^{6}$ Comissão Nacional de Energia Nuclear, CNEN, Rio de Janeiro, RJ, Brazil \\ (*) Corresponding author: E-mail: cotta@mecanica.coppe.ufrj.br
}

\begin{abstract}
The Generalized Integral Transform Technique (GITT) is reviewed as a hybrid numericalanalytical approach for fluid flow problems, with or without heat and mass transfer, here with emphasis on the literature related to flow problems formulated through the full Navier-Stokes equations. A brief overview of the integral transform methodology is first provided for a general nonlinear convection-diffusion problem. Then, different alternatives of eigenfunction expansion strategies are discussed in the integral transformation of problems for which the fluid flow model is either based on the primitive variables or the streamfunction-only formulations, as applied to both steady and transient states. Representative test cases are selected to illustrate the different eigenfunction expansion approaches, with convergence being analyzed for each situation. In addition, fully converged integral transform results are critically compared to previously reported simulations obtained from traditional purely discrete methods.
\end{abstract}

\section{KEYWORDS}

Integral transforms, laminar flow, Navier-Stokes equations, heat and mass transfer, single domain formulation, hybrid methods, eigenfunction expansions. 


\section{INTRODUCTION}

Integral transforms have been widely employed in the solution of differential equations, though their usefulness is not limited to this purpose. It is recognized that Leonhard Euler has in fact introduced the concept of integral transforms in handling second order differential equations [1], first in 1763 for a specific differential equation, and later on in 1769 when the treatment was more systematic and complete [1]. On the other hand, Fourier in his 1822 treatise [2] advanced the idea of Separation of Variables, so as to handle and interpret the solutions of the newly derived heat conduction equation, after proposing the constitutive equation known as Fourier's law. His work provided the modern mathematical theory of heat conduction, but also introduced the well-known Fourier series and Fourier transforms. However, it appears that it was in the work of Acad. N.S. Koshlyakov [3], that the integral transform method gained a more general formalism based on eigenfunction expansions and was first extended to handle linear partial differential equations in finite media with nonhomogeneous terms, either on the main equation or in the boundary conditions, as described in his textbook [4]. This concept of a more general integral transform approach based on eigenfunctions from Sturm-Liouville eigenvalue problems was further explored by Koshlyakov and co-workers [5] and Eringen [6], among others. In the 60's, Luikov [7], Mikhailov [8], and Ozisik [9] made some of the most fundamental contributions for the full establishment of this analytical approach in the heat and mass transfer field. The consolidation of these ideas was systematically presented in the compendium of Mikhailov and Ozisik [10] of 1984, which organized the integral transform analysis of heat and mass diffusion into seven fairly wide classes of linear problems.

The classical integral transform method, despite being applicable to various classes of linear problems, as discussed above, finds limitations that were recognized in attempts of solving problems, still linear but with time-dependent equation or boundary conditions coefficients $[11,12]$, when only approximate analytical solutions were offered for such non-transformable problems. Following this same path, a hybrid numerical-analytical extension was proposed, first in the realm of moving boundary problems [13], and soon after, revisiting the time-dependent coefficients formulations $[14,15]$. This hybrid approach kept along the years the same name of Generalized Integral Transform Technique (GITT), first proposed in [11], and involved the complete solution of the coupled transformed problem, based on the numerical solution of a truncated version of the transformed system of ordinary differential equations. In a relatively short period of time it was 
extended to different classes of problems, including nonlinear diffusion and convection-diffusion [16-19] and irregular domains in parabolic and elliptic formulations [20-23]. It would not take long for the GITT to be challenged by the solution of fluid flow problems governed either by the boundary layer equations or the Navier-Stokes equations [24,25]. Since then, the hybrid method was progressively extended and new classes of problems and applications have been dealt with, and it has been reviewed at different stages and sources [26-34].

The integral transform analysis of fluid flow problems governed by the Navier-Stokes equations has required the proposition of new eigenfunction expansions, other than those normally employed in diffusion or convection-diffusion problems, directly derived from the general Sturm-Liouville eigenvalue problem. Along the years, in the present methodological context, the Navier-Stokes equations have been mostly dealt with in the streamfunction-only formulation [25,35-46], and less frequently in the primitive variables formulation [47,48]. In two-dimensional problems, the streamfunction formulation offers the advantages of automatically satisfying the continuity equation and eliminating the pressure field. In addition, it has been observed through the various applications considered, that the fourth order biharmonic-type eigenvalue problem which is naturally preferred for the eigenfunction expansion in this formulation, results in improved convergence rates in comparison with the previously proposed expansions based on the primitive variables formulation. However, the extension of this concept to three-dimensional flows, leading to vector and scalar potentials, has been shown to be less advantageous when dealt with by the same hybrid approach [49]. Nevertheless, the integral transform method under the two-dimensional streamfunction formulation has been applied to various classes of problems, including cavity and channel flows, rectangular and cylindrical geometries, regular and irregular domains, laminar and turbulent flows, steady and transient states, natural and forced convection, as well as on magnetohydrodynamics [25,35-46]. The integral transformation of the streamfunction formulation is the first one to be here reviewed, for both steady and transient state situations, in light of its popularity among the contributions that employed this hybrid approach so far.

With respect to the primitive variables formulation, which has been more extensively employed in solving the boundary layer equations [50-54], it is worthwhile mentioning first the approach based on the proposition of a Poisson equation for the pressure field [47] and, more recently, based on the manipulation of the momentum equations to eliminate the pressure field [48], but without recalling the streamfunction definition, while the continuity equation is used to determine one of the 
velocity vector components. This second approach is also here reviewed in view of its straightforward extension to the three-dimensional situation.

The present review is focused on the GITT solution of fluid flow problems governed by the Navier-Stokes equations, with or without heat or mass transfer, represented by the associated energy or species conservation equations. This particular emphasis is here chosen in light of recent progresses that are allowing for further generalization of this hybrid method. First, a single domain reformulation strategy has been successfully employed in a number of problems involving heterogeneous media and complex geometries [55-59], with recent extensions to the solution of fluid flow and heat or mass transfer within channels and cavities partially filled with a saturated porous medium [60-63]. Second, a novel interpretation of the eigenfunction expansion proposal in handling the Navier-Stokes equations [60], has unified the treatment of the two- and threedimensional primitive variables formulations into a vector eigenfunction expansion representing all velocity components with one set of transformed potentials and an appropriately chosen vector eigenfunction basis. Through this new interpretation, the velocity vector field can be represented considering the influence of an infinite number of vortices disturbing a base flow. This proposition automatically recovers the streamfunction formulation as a special case for the two-dimensional situation. The combination of these two novel concepts allows for a more straighforward handling of transient flows within complex geometries, such as for instance, in analyzing flow instabilities effects in convective heat and mass transfer problems within porous or partially porous media, previously considered in different physical situations [64-70].

\section{THE GENERALIZED INTEGRAL TRANSFORM TECHNIQUE}

The Generalized Integral Transform Technique (GITT) [26-34], based on the classical integral transform method [10], provides a hybrid numerical-analytical nature to the eigenfunction expansion approach, yielding error-controlled solutions to a large number of linear and nonlinear convection-diffusion problems. The basic steps in the GITT algorithm can be summarized as follows [71,72]:

a) Select an analytical filtering solution to improve convergence behavior of the eigenfunction expansions, if required. Filtering to achieve homogeneous boundary conditions and/or reduce the importance of equation source terms is often helpful; 
b) Choose the associated eigenvalue problem, which should desirably incorporate characteristic linear behaviors of the original problem formulation represented by the coefficients of the differential operators. Either diffusive or convective eigenvalue problems [73] may be adopted. More recently, nonlinear eigenvalue problems have also been employed with marked improvement on convergence $[33,74]$;

c) Develop the integral transform pair and obtain the transform and inversion, that will define the transformation operation and the explicit recovering of the potential;

d) Solve the eigenvalue problem, either in analytical form and symbolic computation, or through the GITT approach itself, transforming the chosen differential eigenvalue problem into an algebraic one $[23,26]$. A convergence acceleration strategy, based on integral balances, has been recently advanced in handling eigenvalue problems through the GITT [75];

e) Integral transform the original PDE and obtain the transformed differential system, which shall be an ODE system for a total transformation, when all the independent variables are eliminated except one. It can result in an initial value problem, for a parabolic or hyperbolic formulation, or in a boundary value problem, for an elliptic formulation. A partial transformation [72] may also be applied, as first proposed in [76], when two independent variables are kept in the transformed system, yielding a partial differential transformed system;

f) Compute transformed system coefficients, which are integrals, single or multiple, involving the eigenfunctions. When analytical expressions are not obtainable through symbolic computation [77], there is a marked advantage in promoting semi-analytical integrations, when the oscillatory nature of the eigenfunctions is analytically handled, while the non-oscillatory portion of the integrand is approximated by piecewise polynomials [71,72];

g) Solve the transformed system, either numerically or analytically, when feasible. This main numerical task requires handling the resulting coupled ODE or PDE system for the transformed potentials, which needs to be truncated to a sufficiently large order for numerical purposes. Reliable automatic solvers are readily available for the numerical solution of stiff ODE systems, and even for one-dimensional systems of PDEs with adaptive remeshing, such as in the routine NDSolve of the Mathematica system [77];

h) Recall inversion formula to analytically reconstruct the hybrid solution of the desired potential. At this point, the inversion formula can be employed for accuracy testing, once the intermediate tasks were error controlled, and then allowing for best selection of the transformed 
system truncation orders. Error estimates are then automatically provided at any desired position and time.

In order to illustrate the basic steps above, let us consider a general nonlinear convectiondiffusion problem of $n$ coupled potentials, $T_{k}(\mathbf{x}, t)$, defined in the region $V$ with boundary surface $S$ :

$$
w_{k}(\mathbf{x}) L_{t, k} T_{k}(\mathbf{x}, t)=L_{\mathbf{x}, k} T_{k}(\mathbf{x}, t)+G_{k}(\mathbf{x}, t, \mathbf{T}), \quad \mathbf{x} \in V, \quad t_{0}<t<t_{1}, \quad k=1,2, \ldots, n
$$

Equation (1a) is already written in a way that all the coupling and nonlinear terms are collapsed into the source terms, $G_{k}(\mathbf{x}, t, \mathbf{T})$, eventually also including convective terms, while the remaining operators retain representative information with selected linear coefficients. Therefore, the $\mathbf{x}$ operator, $L_{\mathbf{x}, k}$, for this diffusion or convection-diffusion problem, may be written as:

$$
L_{\mathbf{x}, k}=\nabla \cdot\left(K_{k}(\mathbf{x}) \nabla T_{k}(\mathbf{x}, t)\right)-d_{k}(\mathbf{x}) T_{k}(\mathbf{x}, t)
$$

which includes the diffusion and linear dissipation terms. The $t$ operator, $L_{t, k}$, for a parabolic formulation may be given by,

$$
L_{k, t} \equiv \frac{\partial}{\partial t}
$$

while for an elliptic or hyperbolic formulation is written as

$$
L_{k, t} \equiv-a_{k}(t) \frac{\partial}{\partial t}\left[b_{k}(t) \frac{\partial}{\partial t}\right]
$$

The source term, including nonlinear convective terms, if pertinent, becomes:

$$
G_{k}(\mathbf{x}, t, \mathbf{T})=-\mathbf{u}(\mathbf{x}, t, \mathbf{T}) \cdot \nabla T_{k}(\mathbf{x}, t)+g_{k}(\mathbf{x}, t, \mathbf{T})
$$

with initial or boundary conditions in the $t$ variable given, respectively, by 


$$
\begin{gathered}
T_{k}\left(\mathbf{x}, t_{0}\right)=f_{k}(\mathbf{x}), \quad \mathbf{x} \in V, \text { for the parabolic problem } \\
T_{k}\left(\mathbf{x}, t_{0}\right)=f_{k}(\mathbf{x}),\left.\quad \frac{\partial T_{k}(\mathbf{x}, t)}{\partial t}\right|_{t=t_{0}}=h_{k}(\mathbf{x}), \quad \mathbf{x} \in V, \text { for the hyperbolic problem } \\
{\left[\lambda_{k, l}(t)+(-1)^{l+1} \gamma_{k, l}(t) \frac{\partial}{\partial t}\right] T_{k}(\mathbf{x}, t)=f_{k, l}(\mathbf{x}), \text { at } t=t_{l}, l=0,1, \mathbf{x} \in V, \text { for the ellliptic problem }}
\end{gathered}
$$

and the boundary conditions in the remaining coordinates

$$
\left[\alpha_{k}(\mathbf{x})+\beta_{k}(\mathbf{x}) K_{k}(\mathbf{x}) \frac{\partial}{\partial \mathbf{n}}\right] T_{k}(\mathbf{x}, t)=\phi_{k}(\mathbf{x}, t, \mathbf{T}), \mathbf{x} \in S, t_{0}<t<t_{1}
$$

where $\mathbf{n}$ denotes the outward-drawn normal to the surface $S$, and the potentials vector is given by

$$
\mathbf{T}=\left\{T_{1}, T_{2}, \ldots, T_{k}, \ldots, T_{n}\right\}^{T}
$$

Equations (1) do not include all of the problem formulations of interest in heat and fluid flow analysis, but are sufficiently general to illustrate the formalism in the GITT. The first step in the integral transform solution of Eqs. (1) is then the proposition of a filtering solution, so as to reduce the importance of the non-homogeneities characterized by the equations and boundary conditions source terms, and their influence on slowing down the eigenfunction expansions convergence rates. Therefore, in general terms, the filter can be written as:

$$
T_{k}(\mathbf{x}, t)=T_{k}^{*}(\mathbf{x}, t)+T_{k, F}(\mathbf{x} ; t)
$$

where the filtering solution, $T_{k, F}(\mathbf{x}, t)$, accounts at least partially for the information within the source terms, $G_{k}(\mathbf{x}, t, \mathbf{T})$ and $\phi_{k}(\mathbf{x}, t, \mathbf{T})$. Then, the resulting filtered potentials should obey the following problem formulation:

$$
w_{k}(\mathbf{x}) L_{t, k} T_{k}^{*}(\mathbf{x}, t)=L_{\mathbf{x}, k} T_{k}^{*}(\mathbf{x}, t)+G_{k}^{*}\left(\mathbf{x}, t, \mathbf{T}^{*}\right), \quad \mathbf{x} \in V, \quad t_{0}<t<t_{1}, \quad k=1,2, \ldots, n
$$


with the filtered source term

$$
G_{k}^{*}\left(\mathbf{x}, t, \mathbf{T}^{*}\right)=G_{k}(\mathbf{x}, t, \mathbf{T})-w_{k}(\mathbf{x}) L_{t, k} T_{k, F}(\mathbf{x}, t)+L_{\mathbf{x}, k} T_{k, F}(\mathbf{x}, t)
$$

and filtered initial and boundary conditions as

$$
\begin{gathered}
T_{k}^{*}\left(\mathbf{x}, t_{0}\right)=f_{k}^{*}(\mathbf{x}) \equiv f_{k}(\mathbf{x})-T_{k, F}\left(\mathbf{x}, t_{0}\right), \quad \mathbf{x} \in V, \text { for the parabolic problem } \\
T_{k}^{*}\left(\mathbf{x}, t_{0}\right)=f_{k}^{*}(\mathbf{x}),\left.\quad \frac{\partial T_{k}^{*}(\mathbf{x}, t)}{\partial t}\right|_{t=t_{0}}=h_{k}^{*}(\mathbf{x}) \equiv h_{k}(\mathbf{x})-\left.\frac{\partial T_{k, F}(\mathbf{x}, t)}{\partial t}\right|_{t=t_{0}}, \quad \mathbf{x} \in V, \\
{\left[\lambda_{k, l}(t)+(-1)^{l+1} \gamma_{k, l}(t) \frac{\partial}{\partial t}\right] T_{k}^{*}(\mathbf{x}, t)=f_{k, l}^{*}(\mathbf{x}) \equiv f_{k, l}(\mathbf{x})-\left[\lambda_{k, l}(t)+(-1)^{l+1} \gamma_{k, l}(t) \frac{\partial}{\partial t}\right] T_{k, F}(\mathbf{x}, t),} \\
\text { at } t=t_{l}, l=0,1, \mathbf{x} \in V
\end{gathered}
$$

for the ellliptic problem

and the boundary conditions in the remaining coordinates

$$
\begin{array}{r}
{\left[\alpha_{k}(\mathbf{x})+\beta_{k}(\mathbf{x}) K_{k}(\mathbf{x}) \frac{\partial}{\partial \mathbf{n}}\right] T_{k}^{*}(\mathbf{x}, t)=\phi_{k}^{*}\left(\mathbf{x}, t, \mathbf{T}^{*}\right) \equiv \phi_{k}(\mathbf{x}, t, \mathbf{T})-\left[\alpha_{k}(\mathbf{x})+\beta_{k}(\mathbf{x}) K_{k}(\mathbf{x}) \frac{\partial}{\partial \mathbf{n}}\right] T_{k, F}(\mathbf{x}, t),} \\
\mathbf{x} \in S, t_{0}<t<t_{1}
\end{array}
$$

Equations (3) were obtained from application of the linear filtering solution of Eq. (2), but other more involved filtering schemes have been proposed, including recursive filtering, local instantaneous filtering, and implicit (or nonlinear) filters [71,72], which shall not be reviewed here for the sake of brevity.

Following the formalism in the GITT [26-34,71,72], an eigenvalue problem is chosen that provides the base for the eigenfunction expansion. Here, when Eqs. (1) were written, this choice was already implicitly made, since characteristic linear coefficients have been identified to describe the differential operators. Therefore, the following eigenvalue problem is considered:

$$
\begin{gathered}
\nabla \cdot\left(K_{k}(\mathbf{x}) \nabla \psi_{k i}(\mathbf{x})\right)+\left[\mu_{k i}^{2} w_{k}(\mathbf{x})-d_{k}(\mathbf{x})\right] \psi_{k i}(\mathbf{x})=0, \quad \mathbf{x} \in V \\
{\left[\alpha_{k}(\mathbf{x})+\beta_{k}(\mathbf{x}) K_{k}(\mathbf{x}) \frac{\partial}{\partial \mathbf{n}}\right] \psi_{k i}(\mathbf{x})=0, \quad \mathbf{x} \in S}
\end{gathered}
$$


Again, more involved eigenvalue problems can be proposed in specific situations, towards improved convergence of the eigenfunction expansions, such as in coupled problems, convective eigenvalue problems, or even nonlinear Sturm Liouville problems, however these alternatives shall not be examined in the present context. Problem (4), with the associated orthogonality property of the eigenfuncionts, $\psi_{k i}(\mathbf{x})$, permits the derivation of the following transform-inverse pair:

$$
\begin{array}{cc}
\bar{T}_{k i}(t)=\int_{V} w_{k}(\mathbf{x}) \tilde{\psi}_{k i}(\mathbf{x}) T_{k}^{*}(\mathbf{x}, t) \mathrm{d} V, & \text { transform } \\
T_{k}^{*}(\mathbf{x}, t)=\sum_{i=1}^{\infty} \tilde{\psi}_{k i}(\mathbf{x}) \bar{T}_{k, i}(t), & \text { inverse }
\end{array}
$$

The symmetric kernels $\tilde{\psi}_{k i}(\mathbf{x})$ and the norms are given by:

$$
\tilde{\psi}_{k i}(\mathbf{x})=\frac{\psi_{k i}(\mathbf{x})}{\sqrt{N_{k i}}} ; \quad N_{k i}=\int_{\mathrm{v}} w_{k}(\mathbf{x}) \psi_{k i}^{2}(\mathbf{x}) \mathrm{d} v
$$

The integral transformation of the partial differential equations (3.a) is then performed, making use of the operator $\int_{V} \tilde{\psi}_{k i}(\mathbf{x})(.) \mathrm{d} V$, to yield the transformed system, after manipulation of the boundary conditions, Eqs.(3.g) and (4.b):

$$
L_{k, t} \bar{T}_{k i}(t)+\mu_{k i}^{2} \bar{k}_{k i}(t)=\bar{g}_{k i}(t, \overline{\mathbf{T}}), i=1,2, \ldots, \quad t_{0}<t<t_{1}, \quad k=1,2, \ldots, n
$$

where the coupling transformed source terms include the influence of the convective terms and of the equation and boundary condition source terms as

$$
\bar{g}_{k i}(t, \overline{\mathbf{T}})=\int_{V} \tilde{\psi}_{k i}(\mathbf{x}) G_{k}^{*}\left(\mathbf{x}, t, \mathbf{T}^{*}\right) d V+\int_{S} \phi_{k}^{*}\left(\mathbf{x}, t, \mathbf{T}^{*}\right)\left[\frac{\tilde{\psi}_{k i}(\mathbf{x})-K_{k}(\mathbf{x}) \frac{\partial \tilde{\psi}_{k i}(\mathbf{x})}{\partial \mathbf{n}}}{\alpha_{k}(\mathbf{x})+\beta_{k}(\mathbf{x})}\right] d s
$$

Similarly, the initial or boundary conditions in the $t$ variable are integral transformed with the operator $\int_{V} w_{k}(\mathbf{x}) \tilde{\psi}_{k i}(\mathbf{x})(.) \mathrm{d} V$, to provide:

$$
\bar{T}_{k i}\left(t_{0}\right)=\bar{f}_{k i} \equiv \int_{V} w_{k}(\mathbf{x}) \tilde{\psi}_{k i}(\mathbf{x}) f_{k}^{*}(\mathbf{x}) d V, \text { for the parabolic problem }
$$




$$
\begin{gathered}
\bar{T}_{k i}\left(t_{0}\right)=\bar{f}_{k i} \equiv \int_{V} w_{k}(\mathbf{x}) \tilde{\psi}_{k i}(\mathbf{x}) f_{k}^{*}(\mathbf{x}) d V,\left.\frac{d \bar{T}_{k i}(t)}{d t}\right|_{t=t_{0}}=\bar{h}_{k i} \equiv \int_{V} w_{k}(\mathbf{x}) \tilde{\psi}_{k i}(\mathbf{x}) h_{k}^{*}(\mathbf{x}) d V \\
\quad \text { for the hyperbolic problem } \quad \text { (6.d,e }) \\
{\left[\lambda_{k, l}(t)+(-1)^{l+1} \gamma_{k, l}(t) \frac{d}{d t}\right] \bar{T}_{k i}(t)=\bar{f}_{k, l i} \equiv \int_{V} w_{k}(\mathbf{x}) \tilde{\psi}_{k i}(\mathbf{x}) f_{k, l}^{*}(\mathbf{x}) d V, \text { at } t=t_{l}, l=0,1} \\
\text { for the elliptic problem }
\end{gathered}
$$

The transformed ordinary differential system, Eqs. (6), is either an initial value problem, for parabolic or hyperbolic formulations, or a boundary value problem for the elliptic formulation, and it is unlikely that analytical solutions can be obtained for such a general nonlinear situation. However, reliable solvers for ODE's are readily available to provide numerical solutions with user prescribed accuracy, such as the routine NDSolve in the Mathematica platform [77]. After numerical integration of the ODE system along the $t$ variable, the inverse formula, Eq. (5b), is recalled to reconstruct the filtered potentials in analytical form.

This hybrid approach has been extensively used as a benchmarking tool, in the verification of more general and flexible numerical codes, but also as a production tool itself, especially in highly intensive computational jobs and/or very precision demanding applications. An open source general purpose symbolic-numerical code was developed, coined as UNIT code (Unified Integral Transforms) [71,72], and implemented in the Mathematica platform [77]. It offers all of the basic analytical and numerical steps in the GITT implementation, allowing for a more straightforward utilization of this methodology. The reader should refer to the recent contributions on the UNIT code for details on the algorithm implementation, both on the total and partial transformation schemes [71,72].

\section{STREAMFUNCTION AND PRIMITIVE VARIABLES FORMULATIONS}

The present section illustrates the application of the GITT in solving the Navier-Stokes equations, either on the streamfunction-only or in the primitive variables formulations. In the first example, it has been chosen to illustrate the solution of the steady state Navier-Stokes equations written in terms of the streamfunction in a cylindrical geometry, representing laminar flow development within a concentric annular tube with rotating inner wall. The second example deals with transient magnetohydrodynamics and natural convection in a rectangular cavity, again in the streamfunction-only formulation, while in the third example, the classical lid driven cavity flow problem is considered with a transient moving lid, handled directly on the primitive variables 
formulation. In the sequence, section 4 presents the recently introduced concept of a vector eigenfunction expansion, which unifies the treatment of two and three-dimensional problems in the primitive variables formulation. Finally, in section 5 , a few results are provided to demonstrate the convergence behavior of the proposed eigenfunction expansions in each case.

\subsection{Streamfunction-only Formulation}

\subsubsection{Steady state}

This example deals with laminar flow inside an annular duct with a rotating inner wall, as illustrated in Fig. 1 [78,79]. A Newtonian fluid enters the duct with a uniform velocity profile $u_{0}$ aligned with the axial direction. The annular duct has inner and outer radii $r_{i}$ and $r_{o}$, respectively. The inner cylinder rotates with an angular velocity $\omega$, whereas the outer cylinder remains at rest. The flow is considered to be steady, laminar, incompressible, and axisymmetric, with constant physical properties.

The flow in the annular region is represented by the continuity and the Navier-Stokes equations in the cylindrical coordinates system. The continuity equation is automatically satisfied when the streamfunction-only formulation is adopted, which is written in terms of the streamfunction and the tangential velocity component, in dimensionless form, as [79]:

$$
\begin{aligned}
& \frac{\partial^{4} \psi}{\partial \mathrm{r}^{4}}-\frac{2}{\mathrm{r}} \frac{\partial^{3} \psi}{\partial \mathrm{r}^{3}}+\frac{3}{\mathrm{r}^{2}} \frac{\partial^{2} \psi}{\partial \mathrm{r}^{2}}-\frac{3}{\mathrm{r}^{3}} \frac{\partial \psi}{\partial \mathrm{r}}+2 \frac{\partial^{4} \psi}{\partial \mathrm{r}^{2} \partial \mathrm{z}^{2}}-\frac{2}{\mathrm{r}} \frac{\partial^{3} \psi}{\partial \mathrm{r} \partial \mathrm{z}^{2}}+\frac{\partial^{4} \psi}{\partial \mathrm{z}^{4}}= \\
& \frac{\operatorname{Re}}{2(1-\gamma)}\left[\frac{1}{\mathrm{r}} \frac{\partial \psi}{\partial \mathrm{z}}\left(\frac{\partial^{3} \psi}{\partial \mathrm{r}^{3}}-\frac{1}{\mathrm{r}} \frac{\partial^{2} \psi}{\partial \mathrm{r}^{2}}+\frac{1}{\mathrm{r}^{2}} \frac{\partial \psi}{\partial \mathrm{r}}+\frac{\partial^{3} \psi}{\partial \mathrm{r} \partial \mathrm{z}^{2}}\right)-\frac{1}{\mathrm{r}} \frac{\partial \psi}{\partial \mathrm{r}}\left(\frac{\partial^{3} \psi}{\partial \mathrm{r}^{2} \partial \mathrm{z}}-\frac{1}{\mathrm{r}} \frac{\partial^{2} \psi}{\partial \mathrm{r} \partial \mathrm{z}}+\frac{\partial^{3} \psi}{\partial \mathrm{z}^{3}}\right)\right. \\
& \left.-\frac{2}{\mathrm{r}^{2}} \frac{\partial \psi}{\partial \mathrm{z}}\left(\frac{\partial^{2} \psi}{\partial \mathrm{r}^{2}}-\frac{1}{\mathrm{r}} \frac{\partial \psi}{\partial \mathrm{r}}+\frac{\partial^{2} \psi}{\partial \mathrm{z}^{2}}\right)-2 \xi^{2} \mathrm{v}_{\theta} \frac{\partial \mathrm{v}_{\theta}}{\partial \mathrm{z}}\right] \\
& \frac{1}{\mathrm{r}} \frac{\partial}{\partial \mathrm{r}}\left(\mathrm{r} \frac{\partial \mathrm{v}_{\theta}}{\partial \mathrm{r}}\right)-\frac{\mathrm{v}_{\theta}}{\mathrm{r}^{2}}+\frac{\partial^{2} \mathrm{v}_{\theta}}{\partial \mathrm{z}^{2}}=\frac{\mathrm{Re}}{2(1-\gamma)}\left[\frac{1}{\mathrm{r}} \frac{\partial \psi}{\partial \mathrm{z}}\left(\frac{\partial \mathrm{v}_{\theta}}{\partial \mathrm{r}}+\frac{\mathrm{v}_{\theta}}{\mathrm{r}}\right)-\frac{1}{\mathrm{r}} \frac{\partial \psi}{\partial \mathrm{r}} \frac{\partial \mathrm{v}_{\theta}}{\partial \mathrm{z}}\right]
\end{aligned}
$$

The boundary conditions are given by:

$$
\psi(\gamma, \mathrm{z})=\mathrm{C}_{1} ; \quad \frac{\partial \psi(\gamma, \mathrm{z})}{\partial \mathrm{r}}=0 ; \quad \mathrm{v}_{\theta}(\gamma, \mathrm{z})=1
$$




$$
\begin{aligned}
& \psi(1, \mathrm{z})=\mathrm{C}_{2} ; \quad \frac{\partial \psi(1, \mathrm{z})}{\partial \mathrm{r}}=0 ; \quad \mathrm{v}_{\theta}(1, \mathrm{z})=0 \\
& \psi(\mathrm{r}, 0)=\mathrm{C}_{1}-\left(\mathrm{r}^{2}-\gamma^{2}\right) / 2 ; \quad \frac{\partial^{2} \psi(\mathrm{r}, 0)}{\partial \mathrm{z}^{2}}=0 ; \quad \mathrm{v}_{\theta}(\mathrm{r}, 0)=0 \\
& \psi(\mathrm{r}, \infty)=\psi_{\infty}(\mathrm{r}) ; \quad \frac{\partial \psi(\mathrm{r}, \infty)}{\partial \mathrm{z}}=0 ; \quad \mathrm{v}_{\theta}(\mathrm{r}, \infty)=\mathrm{v}_{\theta, \infty}(\mathrm{r})
\end{aligned}
$$

where, $\psi_{\infty}(\mathrm{r})$ and $\mathrm{v}_{\theta, \infty}(\mathrm{r})$ are the values of the streamfunction and the tangential velocity component in the fully developed region. Also, $\mathrm{C}_{1}$ and $\mathrm{C}_{2}$ are the values of the streamfunction at the inner and outer cylinder walls, respectively. These quantities are defined as:

$$
\begin{gathered}
\psi_{\infty}(r)=C_{2}-\frac{2}{\beta}\left[r_{m}^{2}\left(r^{2} \ln r-\gamma^{2} \ln r\right)-\frac{\left(r^{2}-\gamma^{2}\right)}{4}\left(2 r_{m}^{2}-2+r^{2}+\gamma^{2}\right)\right] ; C_{1}=0 ; \quad C_{2}=-\frac{\left(1-\gamma^{2}\right)}{2} \text { (7.o-q) } \\
v_{\theta, \infty}(r)=\frac{\gamma}{\left(1-\gamma^{2}\right)}\left(\frac{1}{r}-r\right) ; \beta=1+\gamma^{2}-2 r_{m}^{2} ; \quad r_{m}=\left\{\frac{\left(1-\gamma^{2}\right)}{2 \ln (1 / \gamma)}\right\}^{1 / 2}
\end{gathered}
$$

The dimensionless groups employed in the above formulation are:

$$
\begin{aligned}
& \mathrm{r}=\frac{\mathrm{r}^{*}}{\mathrm{r}_{\mathrm{o}}} ; \mathrm{z}=\frac{\mathrm{z}^{*}}{\mathrm{r}_{\mathrm{o}}} ; \mathrm{v}_{\mathrm{z}}=\frac{\mathrm{v}_{\mathrm{z}}^{*}}{\mathrm{u}_{\mathrm{o}}} ; \mathrm{v}_{\mathrm{r}}=\frac{\mathrm{v}_{\mathrm{r}}^{*}}{\mathrm{u}_{\mathrm{o}}} ; \mathrm{v}_{\theta}=\frac{\mathrm{v}_{\theta}^{*}}{\omega \mathrm{r}_{\mathrm{i}}} ; \mathrm{p}=\frac{\mathrm{p}^{*}}{\rho \mathrm{u}_{\mathrm{o}}^{2}} ; \gamma=\frac{\mathrm{r}_{\mathrm{i}}}{\mathrm{r}_{\mathrm{o}}} \\
& \operatorname{Re}=\frac{2\left(\mathrm{r}_{\mathrm{o}}-\mathrm{r}_{\mathrm{i}}\right) \mathrm{u}_{0}}{\mathrm{v}} ; \xi=\frac{\omega \mathrm{r}_{\mathrm{i}}}{\mathrm{u}_{\mathrm{o}}}=\frac{1}{\operatorname{Re}} \sqrt{\frac{2 \mathrm{Ta}(1+\gamma)}{(1-\gamma)}} ; \mathrm{Ta}=\frac{2\left(\omega \mathrm{r}_{\mathrm{i}}\right)^{2}\left(\mathrm{r}_{\mathrm{o}}-\mathrm{r}_{\mathrm{i}}\right)^{3}}{v^{2}\left(\mathrm{r}_{\mathrm{o}}+\mathrm{r}_{\mathrm{i}}\right)}
\end{aligned}
$$

where the main parameters in Eqs. (8) are the Reynolds number (Re), the Taylor number (Ta), the rotation parameter $(\xi)$, and the radii ratio $(\gamma)$. The streamfunction is related to the radial and axial velocity components, $\mathrm{v}_{\mathrm{r}}$ and $\mathrm{vz}$, as:

$$
\mathrm{v}_{\mathrm{r}}=\frac{1}{\mathrm{r}} \frac{\partial \psi}{\partial \mathrm{z}} ; \quad \mathrm{v}_{\mathrm{z}}=-\frac{1}{\mathrm{r}} \frac{\partial \psi}{\partial \mathrm{r}}
$$


In solving Eqs. (1), filtering is applied in order to improve the computational performance, by eliminating the non-homogeneity of the boundary conditions in the r-direction, since this is the chosen coordinate for elimination through the integral transformation process. This filter is given as:

$$
\mathrm{v}_{\theta}(\mathrm{r}, \mathrm{z})=\mathrm{v}_{\theta, \infty}(\mathrm{r})+\mathrm{v}_{\theta}^{*}(\mathrm{r}, \mathrm{z}) ; \quad \psi(\mathrm{r}, \mathrm{z})=\psi_{\infty}(\mathrm{r})+\phi(\mathrm{r}, \mathrm{z})
$$

Eigenfunction expansions are then proposed for the streamfunction and tangential velocity fields, by considering a biharmonic-type problem for the streamfunction and a Sturm-Liouville problem for the tangential velocity component, both dependent on the radial direction only. Here, the integral transformation is promoted only in the radial direction, thus leading to a transformed ODE system (boundary value problem) in the $\mathrm{z}$ direction. The solution of the coupled non-linear ordinary differential equations for the transformed potentials is obtained through the DBVPFD subroutine of the IMSL library [80].

\subsubsection{Transient state}

This example considers the influence of a magnetic field in the momentum and heat transfer in transient MHD flow in a square cavity, for conditions of moderate and high Grashof numbers [44, 81-85], as illustrated in Fig. 2. The cavity has an infinite extent along the z-axis, the lower and upper walls are insulated, while the side walls are maintained at different and constant temperatures, namely, the hot $\left(\mathrm{T}_{\mathrm{h}}\right)$ and cold $\left(\mathrm{T}_{\mathrm{c}}\right)$ walls, respectively. The flow is in transient state and the fluid is Newtonian and electrically conductive. Moreover, the fluid properties are considered constant throughout the range of temperatures in the specific example. The temperature difference causes the movement through the onset of the buoyancy force. This term in the momentum equations is modeled using the Boussinesq approximation, with density variation in the body force term only. The fluid is permeated by a constant magnetic field $B_{0}$ applied in the x-direction (from the left to the right wall), which creates a force opposing the buoyancy effect, the Lorentz force, represented by the vector product between the electrical current density and the magnetic field. Therefore, the equations governing the problem are the continuity, Navier-Stokes and energy equations, as well as the electric charges conservation equation, Ohm's Law, and Ampère-Maxwell's law in a moving 
medium. Adopting the streamfunction-only formulation, the problem is written in dimensionless form as [81]:

$$
\begin{gathered}
\frac{\partial\left(\nabla^{2} \psi\right)}{\partial t}+\frac{\partial \psi}{\partial y}\left(\frac{\partial^{3} \psi}{\partial x^{3}}+\frac{\partial^{3} \psi}{\partial x \partial y^{2}}\right)-\frac{\partial \psi}{\partial x}\left(\frac{\partial^{3} \psi}{\partial x^{2} \partial y}+\frac{\partial^{3} \psi}{\partial y^{3}}\right)=\operatorname{Pr} \nabla^{4} \psi-\operatorname{Pr} H a^{2} \frac{\partial^{2} \psi}{\partial x^{2}}-\operatorname{Ra} \operatorname{Pr} \frac{\partial T}{\partial x} \\
\frac{\partial T}{\partial t}+\frac{\partial \psi}{\partial y} \frac{\partial T}{\partial x}-\frac{\partial \psi}{\partial x} \frac{\partial T}{\partial y}=\nabla^{2} T
\end{gathered}
$$

The initial and boundary conditions, in dimensionless form, needed to complete the mathematical formulation, are:

$$
\begin{gathered}
T(x, y, 0)=\psi(x, y, 0)=0 \text { for } t=0 \\
\psi=\frac{\partial \psi}{\partial x}=0 ; \quad T=1 \quad \text { at } \quad x=0 ; \quad \psi=\frac{\partial \psi}{\partial x}=T=0 \quad \text { at } \quad x=1 \\
\psi=\frac{\partial \psi}{\partial y}=\frac{\partial T}{\partial y}=0 \quad \text { at } y=0 ; \quad \psi=\frac{\partial \psi}{\partial y}=\frac{\partial T}{\partial y}=0 \quad \text { at } \quad y=1
\end{gathered}
$$

The usual definition of the streamfunction was adopted, in the form:

$$
u^{*}=\frac{\partial \psi^{*}}{\partial y^{*}} ; \quad v^{*}=-\frac{\partial \psi^{*}}{\partial x^{*}}
$$

together with the following dimensionless quantities:

$$
x=\frac{x^{*}}{L} ; \quad y=\frac{y^{*}}{L} ; \quad \psi=\frac{\psi^{*}}{\alpha_{T}} ; \quad t=\frac{\alpha_{T} t^{*}}{L^{2}} ; \quad T=\frac{T^{*}-T_{c}}{T_{h}-T_{c}}
$$

where the subscript "*” identifies the dimensional variables, $\alpha_{\mathrm{T}}$ is the fluid thermal diffusivity, and $L$ is the length of the cavity. Also, $\mathrm{Ha}, \mathrm{Ra}, \mathrm{Pr}$, and $\mathrm{Gr}$ are the Hartmann, Rayleigh, Prandtl and Grashof numbers, respectively, which are defined as: 


$$
H a=B_{0} L \sqrt{\frac{\sigma}{\mu_{0}}} ; \quad \operatorname{Ra}=\frac{g \beta_{T}\left(T_{h}-T_{c}\right) L^{3}}{\alpha_{T} v} ; \quad \operatorname{Pr}=\frac{v}{\alpha_{T}} ; \quad G r=\frac{g \beta_{T}\left(T_{h}-T_{c}\right) L^{3}}{v^{2}}=\frac{R a}{\operatorname{Pr}}
$$

where $v$ is the kinematic viscosity, $\beta_{T}$ is the coefficient of thermal expansion, $\sigma$ is the electrical conductivity and $\mu_{0}$ is the magnetic permeability in vacuum. In this study, the effects of polarization and magnetization were neglected. The magnetic Reynolds number is considered to be very small, as well as the effects of Joule heating and viscous dissipation. In integral transforming the flow problem, Eq. (11.a), the preferred eigenvalue problem is the biharmonic equation in both space coordinates, $x$ and $y$, as analyzed in [81]. For the temperature problem, Eq. (11.b), the twodimensional Sturm-Liouville equation with constant coefficients is adopted. Then, the transformed system becomes an initial value problem (ODEs system), which has been accurately handled by subroutine DIVPAG of the IMSL Library [80].

\subsection{Primitive Variables Formulation}

Consider the transient two-dimensional Navier-Stokes equations with constant properties for an incompressible flow with negligible body forces, together with the corresponding continuity equation, for the classical lid-driven cavity flow problem in primitive variables formulation. The fluid, initially at rest, has impermeability and no-slip conditions at the walls, except on the top surface where a reference uniform time-variable velocity, $f(t)$, is imposed $[48,86]$. The primitive variables formulation in dimensionless form in the square region $0<y<1$ and $0<x<1$, as represented in Fig. 3, is given by [48]:

$$
\begin{gathered}
\frac{\partial v(x, y, t)}{\partial y}+\frac{\partial u(x, y, t)}{\partial x}=0 \\
\frac{\partial u}{\partial t}+u \frac{\partial u}{\partial x}+v \frac{\partial u}{\partial y}=-\frac{\partial p}{\partial x}+\frac{1}{\operatorname{Re}}\left(\frac{\partial^{2} u}{\partial x^{2}}+\frac{\partial^{2} u}{\partial y^{2}}\right) \\
\frac{\partial v}{\partial t}+u \frac{\partial v}{\partial x}+v \frac{\partial v}{\partial y}=-\frac{\partial p}{\partial y}+\frac{1}{\operatorname{Re}}\left(\frac{\partial^{2} v}{\partial x^{2}}+\frac{\partial^{2} v}{\partial y^{2}}\right)
\end{gathered}
$$

with the following initial and boundary conditions: 


$$
\begin{gathered}
u(x, y, 0)=0 ; \quad u(1, y, t)=0 ; \quad u(0, y, t)=0 ; \quad u(x, 0, t)=0 ; \quad u(x, 1, t)=f(t) \\
v(x, y, 0)=0 ; \quad v(1, y, t)=0 ; \quad v(0, y, t)=0 ; \quad v(x, 0, t)=0 ; \quad v(x, 1, t)=0
\end{gathered}
$$

It has here been chosen to illustrate the integral transformation alternative path of handling the primitive variables Navier-Stokes equations, as proposed in [48]. The idea is to manipulate the momentum equations, such as in the case of the streamfunction formulation, so as to eliminate the pressure field. Then, the continuity equation in Eq. (15.a) is integral transformed and used to express one of the transformed velocity components in terms of the other component. Thus, the first step in the approach consists in eliminating the pressure term from the Navier-Stokes equations, which requires that one differentiates Eq. (15.b) with respect to $y$ and Eq.(15.c) with respect to $x$, and the results are then subtracted, yielding:

$$
\left(\frac{\partial}{\partial y} \frac{\partial u}{\partial t}-\frac{\partial}{\partial x} \frac{\partial v}{\partial t}\right)+\left(u \frac{\partial^{2} u}{\partial y \partial x}+v \frac{\partial^{2} u}{\partial^{2} y}\right)-\left(v \frac{\partial^{2} v}{\partial y \partial x}+u \frac{\partial^{2} v}{\partial x^{2}}\right)=-\frac{1}{\operatorname{Re}} \frac{\partial}{\partial x}\left(\frac{\partial^{2} v}{\partial x^{2}}+\frac{\partial^{2} v}{\partial y^{2}}\right)+\frac{1}{\operatorname{Re}} \frac{\partial}{\partial y}\left(\frac{\partial^{2} u}{\partial x^{2}}+\frac{\partial^{2} u}{\partial y^{2}}\right)
$$

Equations (15.a) and (16), together with initial and boundary conditions, Eqs. (15.d-m), complete the problem statement in terms of the primitive variables formulation, without the pressure terms. Here, second order diffusion-type eigenvalue problems have been preferred, and there is the choice of implementing a total or partial integral transformation scheme. An application will be reported in section 5, for an oscillating lid velocity $f(t)=\cos (\omega \mathrm{t})$, with $\omega=2 \pi / \tau$, where $\omega$ is the oscillating frequency and $\tau$ is the time period [86].

The same procedure can be used for a three-dimensional formulation. In this situation, the resulting system of equations is given by the continuity equation and the other two equations generated in the manipulation of the three momentum equations to eliminate the pressure field, yielding:

$$
\frac{\partial u(x, y, z, t)}{\partial x}+\frac{\partial v(x, y, z, t)}{\partial y}+\frac{\partial w(x, y, z, t)}{\partial z}=0
$$




$$
\begin{aligned}
& \frac{\partial w(x, y, z, t)}{\partial z}\left(\frac{\partial v(x, y, z, t)}{\partial x}-\frac{\partial u(x, y, z, t)}{\partial y}\right)+\frac{\partial w(x, y, z, t)}{\partial y} \frac{\partial u(x, y, z, t)}{\partial z}-\frac{\partial w(x, y, z, t)}{\partial x} \frac{\partial v(x, y, z, t)}{\partial z}+ \\
& u(x, y, z, t)\left(\frac{\partial^{2} u(x, y, z, t)}{\partial y \partial x}-\frac{\partial^{2} v(x, y, z, t)}{\partial^{2} x}\right)+v(x, y, z, t)\left(\frac{\partial^{2} u(x, y, z, t)}{\partial^{2} y}-\frac{\partial^{2} v(x, y, z, t)}{\partial x \partial y}\right)+ \\
& w(x, y, z, t)\left(\frac{\partial^{2} u(x, y, z, t)}{\partial y \partial z}-\frac{\partial^{2} v(x, y, z, t)}{\partial x \partial z}\right)=-\frac{\partial}{\partial y} \frac{\partial u(x, y, z, t)}{\partial t}+\frac{\partial}{\partial x} \frac{\partial v(x, y, z, t)}{\partial t}+ \\
& \frac{1}{\operatorname{Re}} \frac{\partial}{\partial y} \nabla^{2} u(x, y, z, t)-\frac{1}{\operatorname{Re}} \frac{\partial}{\partial x} \nabla^{2} v(x, y, z, t) \\
& \frac{\partial v(x, y, z, t)}{\partial y}\left(\frac{\partial w(x, y, z, t)}{\partial x}-\frac{\partial u(x, y, z, t)}{\partial z}\right)+\frac{\partial v(x, y, z, t)}{\partial z} \frac{\partial u(x, y, z, t)}{\partial y}-\frac{\partial v(x, y, z, t)}{\partial x} \frac{\partial w(x, y, z, t)}{\partial y}+ \\
& u(x, y, z, t)\left(\frac{\partial^{2} u(x, y, z, t)}{\partial z \partial x}-\frac{\partial^{2} w(x, y, z, t)}{\partial^{2} x}\right)+v(x, y, z, t)\left(\frac{\partial^{2} u(x, y, z, t)}{\partial z \partial y}-\frac{\partial^{2} w(x, y, z, t)}{\partial x \partial y}\right)+ \\
& w(x, y, z, t)\left(\frac{\partial^{2} u(x, y, z, t)}{\partial^{2} z}-\frac{\partial^{2} w(x, y, z, t)}{\partial x \partial z}\right)= \\
& -\frac{\partial}{\partial z} \frac{\partial u(x, y, z, t)}{\partial t}+\frac{\partial}{\partial x} \frac{\partial w(x, y, z, t)}{\partial t}+\frac{1}{\operatorname{Re}} \frac{\partial}{\partial z} \nabla^{2} u(x, y, z, t)-\frac{1}{\operatorname{Re}} \frac{\partial}{\partial z} \nabla^{2} w(x, y, z, t)
\end{aligned}
$$

\section{VECTOR EIGENFUNCTION EXPANSION}

Next, recent advances on combining integral transforms with the single domain reformulation strategy and a vector eigenfunction expansion [60] are briefly discussed. The example here considered represents a parallel plate channel partially filled with a porous medium. The model for the fluid flow inside the porous medium is the Darcy-Brinkman, while for the free fluid, is the Navier-Stokes equations for steady state incompressible flow. With the main advantage of accomplishing the coupling between the regions automatically and more conveniently for computational purposes, the single domain formulation is here employed, represented through appropriate space variable coefficients. The problem formulation then becomes [60]:

$$
\begin{gathered}
\nabla \cdot \boldsymbol{u}=0 \\
\rho(\boldsymbol{u} \cdot \nabla) \boldsymbol{u}=-\nabla p+\frac{4}{\operatorname{Re}} \nabla \cdot[\mu \nabla \boldsymbol{u}]-\frac{4 \gamma}{\operatorname{ReDa}} \boldsymbol{u}
\end{gathered}
$$


where $\boldsymbol{u}$ is the dimensionless velocity vector, $\nabla$ is the dimensionless nabla operator, $\rho$ is the dimensionless density, $p$ is the dimensionless pressure field, $\mu$ is the dimensionless dynamic viscosity, $R e$ is the Reynolds number based on the hydraulic diameter, and $D a$ is the Darcy number. The dimensionless quantities are given by:

$$
\boldsymbol{u}=\frac{\boldsymbol{u}^{*}}{u_{0}} ; \nabla=h \nabla^{*} ; \rho=\frac{\rho^{*}}{\rho_{0}} ; p=\frac{p^{*}}{\rho_{0} u_{0}^{2}} ; \mu=\frac{\mu^{*}}{\mu_{0}} ; \operatorname{Re}=\frac{4 \rho_{0} u_{0} h}{\mu_{0}} ; D a=\frac{\kappa}{h^{2}}
$$

where $\boldsymbol{u}^{*}$ is the velocity vector, $u_{0}$ is the uniform entry longitudinal velocity component, $\nabla^{*}$ is the nabla operator, $h$ is half the height of the channel, $\rho^{*}$ is the density, $\rho_{0}$ is the fluid density, $p^{*}$ is the pressure field, $\mu^{*}$ is the viscosity, $\mu_{0}$ is the fluid viscosity, and $\kappa$ is the permeability of the porous medium.

In accordance with the single domain formulation [55-59], the physical properties of Eqs. (18) vary abruptly across the interfaces between different media. Let $V_{f}$ and $V_{p}$ be the regions occupied by the fluid and porous layers, respectively. Employing the effective viscosity, neglecting the inertial terms within the porous medium, and disregarding the dissipative term in the fluid layer, the following definitions are adopted:

$$
\rho=\left\{\begin{array}{l}
1, \text { in } V_{f} \\
0, \text { in } V_{p}
\end{array} ; \quad \mu=\left\{\begin{array}{c}
1, \text { in } V_{f} \\
1 / \varepsilon, \text { in } V_{p}
\end{array} ; \quad \gamma=\left\{\begin{array}{l}
0, \text { in } V_{f} \\
1, \text { in } V_{p}
\end{array} ;\right.\right.\right.
$$

where $\varepsilon$ is the porosity of the porous medium.

The Brinkman viscous correction allows for the imposition of no-slip boundary conditions at the walls. At the entrance, a uniform velocity profile was imposed. The outlet boundary condition is prescribed assuming that the channel is long enough for the flow to be fully developed. Hence, a dimensionless fully developed velocity profile $F(y)$, as detailed in [60], is considered at the outlet. The adopted single domain approach eliminates the need to explicitly specify boundary conditions at the interface between the free fluid and porous layers. Thus, the boundary conditions are given as: 


$$
\begin{gathered}
\boldsymbol{u}(x,-1)=0 ; \quad \boldsymbol{u}(x, 1)=0 \\
\boldsymbol{u}(0, y)=\left[\begin{array}{lll}
f(y) & 0 & 0
\end{array}\right]^{T} ; \boldsymbol{u}\left(x_{o}, 1\right)=\left[\begin{array}{lll}
F(y) & 0 & 0
\end{array}\right]^{T}
\end{gathered}
$$

where $x_{o}$ is the dimensionless channel length and $f$ is the velocity profile at the entry of the channel.

The first step is then the filtering of the velocity vector, in the form:

$$
\boldsymbol{u}(x, y)=\hat{\boldsymbol{u}}(x, y)+\boldsymbol{u}_{f}(x, y), \text { where } \boldsymbol{u}_{f}=\left[\begin{array}{lll}
F(y) & 0 & 0
\end{array}\right]^{T}
$$

The velocity vector field can be determined by taking into account the influence of an infinite number of vortices disturbing a base flow. Then, this novel interpretation of the eigenfunction expansion is introduced to represent the filtered velocity vector as [60]:

$$
\hat{\boldsymbol{u}}(x, y)=\sum_{i=1}^{\infty} \nabla \times\left[\bar{\phi}_{i}(x) \boldsymbol{\Phi}_{i}(y)\right]
$$

Expanding eq. (23) in the Cartesian coordinate system, imposing that the only non-zero component of the base vector $\boldsymbol{\Phi}_{i}$ is in the z-direction, and assuming the linearity of the curl operator holds for the infinite series involved, the following expression is achieved:

$$
\hat{\boldsymbol{u}}(x, y)=\left[\frac{\partial}{\partial y}\left\{\sum_{i=1}^{\infty} \bar{\phi}_{i}(x) \tilde{\varphi}_{i}(y)\right\}-\frac{\partial}{\partial x}\left\{\sum_{i=1}^{\infty} \bar{\phi}_{i}(x) \tilde{\varphi}_{i}(y)\right\} \quad 0\right]^{T}
$$

where $\tilde{\varphi}_{i}$ is taken as the z-axis component of $\boldsymbol{\Phi}_{i}$. Defining the summation within the derivatives in eq. (24) as the streamfunction, the correspondence between the present approach and the streamfunction formulation is fully established. However, the interpretation without recurring to the streamfunction definition allows for a more straightforward generalization to three-dimensional problems, provided that a proper base for the expansion $\left(\boldsymbol{\Phi}_{i}\right)$ is made available. In the single domain framework, the integral transform methodology strongly benefits from the inclusion of the abrupt variation of the physical properties that represent the media transitions. Therefore, an 
extension of the classical biharmonic eigenvalue problem has been preferred in the present situation [60]. This eigenvalue problem with space variable coefficients is itself handled by the GITT by considering a simpler auxiliary eigenvalue problem [26].

Further extension of the physical concept of a base flow disturbed by an infinite number of vortices is envisioned, aiming at the solution of the flow equations in its three-dimensional transient form. Thus, consider the fairly general formulation of the transient Navier-Stokes equations in dimensionless vector form for an incompressible flow given by:

$$
\begin{gathered}
\nabla \cdot \boldsymbol{u}=0, \quad \boldsymbol{x} \in V \\
\frac{\partial \boldsymbol{u}}{\partial t}+\nabla \cdot(\boldsymbol{u} \otimes \boldsymbol{u})=-\nabla p+\frac{1}{\operatorname{Re}} \nabla \cdot[\mu \nabla \boldsymbol{u}], \quad \boldsymbol{x} \in V
\end{gathered}
$$

where $V$ represents the domain occupied by the fluid, $\boldsymbol{u}$ is the dimensionless velocity vector, $p$ is the dimensionless pressure field, $R e$ is the Reynolds number, $\mu$ is the dimensionless viscosity.

Recalling the separation of the flow field into a base flow and an infinite number of disturbing vortices of Eq. (22.a), the latter are represented in a more convenient way for solving Eqs. (25.a,b), as follows:

$$
\hat{\boldsymbol{u}}(\boldsymbol{x}, t)=\sum_{i=1}^{\infty} \bar{u}_{i}(t)\left(\nabla \times \tilde{\boldsymbol{\Phi}}_{i}\right)
$$

Assuming the base flow satisfies the continuity equation (25.a), the definition of Eq. (26) is sufficient to warrant mass conservation likewise in the streamfunction-only formulation, dropping the need to further analyze Eq. (25.a).

A convenient way to assure the convergence of the eigenfunction expansion of eq. (26) would be to impose the orthogonality property to $\left(\nabla \times \tilde{\boldsymbol{\Phi}}_{i}\right)$, while including physical information into it. A straightforward way of accomplishing these requirements is to use a vector eigenvalue problem capable of decoupling system (27.a-h) when $R e \rightarrow 0$ (Stoke's flow). The resulting self-adjoint vector eigenvalue problem is given by [87]:

$$
\nabla \times \nabla \cdot\left[\mu \nabla\left(\nabla \times \tilde{\boldsymbol{\Phi}}_{i}\right)\right]+\eta_{i}^{2}\left(\nabla \times \nabla \times \tilde{\boldsymbol{\Phi}}_{i}\right)=\mathbf{0}
$$


The orthogonality property of the solution to eq. (27) allows for the proposition of a transformed velocity, in the form:

$$
\bar{u}_{i}(t)=\int_{V}\left(\nabla \times \tilde{\boldsymbol{\Phi}}_{i}\right) \cdot \hat{\boldsymbol{u}}(\boldsymbol{x}, t) d v
$$

Equations (26) and (28) form a transform-inverse pair, reproducing the same formalism as in the usual application of the GITT.

Let $\partial V_{e}$ be the boundary of $V$ containing the entry region, $\partial V_{w}$ be the boundary of $V$ containing the wall region, $\partial V_{o}$ be the boundary of $V$ containing the outlet region, where $\partial V=\partial V_{e} \cup \partial V_{w} \cup \partial V_{o}$. Substituting eq. (22.a) into eq. (25.b), applying $\int_{V}\left(\nabla \times \Phi_{i}\right) \cdot \_d v$, using typical boundary conditions for the entry, wall, and outlet regions, and using some vector calculus identities, the following transformed system of ordinary differential equations results:

$$
\frac{d \bar{u}_{i}}{d t}+\frac{\eta_{i}^{2}}{\operatorname{Re}} \bar{u}_{i}(t)+\sum_{k=1}^{\infty} \sum_{j=1}^{\infty} B_{i j k} \bar{u}_{j}(t) \bar{u}_{k}(t)+\sum_{j=1}^{\infty}\left(C_{i j}+D_{i j}\right) \bar{u}_{j}(t)=\bar{g}_{1, i}+\bar{g}_{2, i}
$$

with integral coefficients given by,

$$
\begin{gathered}
B_{i j k}=\int_{\partial V_{o}}\left[\left(\nabla \times \boldsymbol{\Phi}_{j}\right) \cdot \boldsymbol{n}\right]\left\{\left(\nabla \times \boldsymbol{\Phi}_{k}\right) \cdot\left(\nabla \times \boldsymbol{\Phi}_{i}\right)\right\} d s-\int_{V}\left[\left(\nabla \times \boldsymbol{\Phi}_{j}\right) \otimes\left(\nabla \times \boldsymbol{\Phi}_{k}\right)\right]: \nabla\left(\nabla \times \boldsymbol{\Phi}_{i}\right) d v \\
C_{i j}=\int_{\partial V_{o}}\left(\boldsymbol{u}_{f} \cdot \boldsymbol{n}\right)\left[\left(\nabla \times \boldsymbol{\Phi}_{i}\right) \cdot\left(\nabla \times \boldsymbol{\Phi}_{j}\right)\right] d s-\int_{V}\left[\boldsymbol{u}_{f} \otimes\left(\nabla \times \boldsymbol{\Phi}_{j}\right)\right]: \nabla\left(\nabla \times \boldsymbol{\Phi}_{i}\right) d v \\
D_{i j}=\int_{\partial V_{o}}\left[\left(\nabla \times \boldsymbol{\Phi}_{j}\right) \cdot \boldsymbol{n}\right]\left[\boldsymbol{u}_{f} \cdot\left(\nabla \times \boldsymbol{\Phi}_{i}\right)\right] d s-\int_{V}\left[\left(\nabla \times \boldsymbol{\Phi}_{j}\right) \otimes \boldsymbol{u}_{f}\right]: \nabla\left(\nabla \times \boldsymbol{\Phi}_{i}\right) d v \\
\bar{g}_{1, i}=-\int_{\partial V_{e}} \hat{p}_{e}\left(\nabla \times \boldsymbol{\Phi}_{i}\right) \cdot \boldsymbol{n} d s-\int_{\partial V_{o}} \hat{p}_{o}\left(\nabla \times \boldsymbol{\Phi}_{i}\right) \cdot \boldsymbol{n} d s \\
\bar{g}_{2, i}=\int_{V}\left(\nabla \times \boldsymbol{\Phi}_{i}\right) \cdot\left\{\frac{1}{R e} \nabla \cdot\left[\mu \nabla \boldsymbol{u}_{f}\right]-\nabla \cdot\left(\boldsymbol{u}_{f} \otimes \boldsymbol{u}_{f}\right)\right\} d v
\end{gathered}
$$

where $\hat{p}_{e}$ and $\hat{p}_{o}$ are the filtered pressures at the entry and outlet regions, respectively. 
The numerical solution to the transformed problem of eqs. (29.a-f), once inserted into the inverse formula of Eq. (26), allows for recovery of the velocity vector.

\section{RESULTS AND DISCUSSION}

The four solution paths above discussed are now exemplified, with emphasis on the illustration of convergence behavior of the associated eigenfunction expansions and verification with numerical solutions available in the literature or here obtained through commercial numerical platforms.

\subsection{Streamfunction-only Formulation: Steady laminar flow in annular tube with rotation}

Table 1 shows the convergence behavior of the axial velocity component, for the dimensionless axial positions $\mathrm{z}=0.54$ and $\mathrm{z}=2.7$, for $\mathrm{Re}=300$ and $\gamma=0.1$, considering the case with rotation of the inner cylinder for $\xi=1$. It can be observed that all of the velocity field results are fully converged to four significant digits in the range of truncation orders shown for the eigenfunction expansion $(\mathrm{N}<39)$. At the position further downstream convergence is noticeably faster than at the position closer to the channel entrance, at position $\mathrm{z}=0.54$. This is due to the adopted filtering solution which extracts the fully developed velocity profile for large $\mathrm{z}$.

Figure 4 shows the development of the axial velocity component evaluated at different axial positions for $\operatorname{Re}=300$ and $\gamma=0.1$ for the case with rotation of the inner cylinder, considering $\xi=1$. It can be observed that the fully converged results obtained through integral transforms (GITT) have a perfect agreement, in the graph scale, with those computed through the COMSOL Multiphysics platform.

\subsection{Streamfunction-only Formulation: Transient natural convection with MHD flow}

Test cases were analyzed for Grashof numbers equal to $10^{4}$ and $10^{6}$, Hartmann number in the range from $0<\mathrm{Ha}<100$, while the Prandtl number was taken equal to 0.71 in all cases. First, the convergence behavior of the global mean Nusselt number, streamfunction modulus and the dimensionless temperature at the points $(\mathrm{x}=0.1 ; \mathrm{y}=0.1)$ and $(\mathrm{x}=0.9 ; \mathrm{y}=0.9)$ of the cavity, for $\mathrm{t}=0.005$ and for steady state, with $\mathrm{Gr}=10^{4}$ and $\mathrm{Ha}=0$, is shown in Table 2 . The results in this table illustrate the excellent convergence rates both at the beginning of the transient process and at steady state. The global mean Nusselt number converges with fewer terms, while the streamfunction has a 
slower convergence rate. The steady state velocity $\mathrm{x}$-component at the vertical midplane of the cavity $(\mathrm{x}=1 / 2)$, for different values of $\mathrm{Ha}$ from 0 to 50 and $\mathrm{Gr}=10^{4}$, is shown in Figure 5.a, comparing the present results obtained by the GITT approach with those in [82] that employed a mesh-free kernel approximation technique based on radial basis functions (RBFs). For the lowest Hartmann number analyzed $(\mathrm{Ha}=0)$, the behavior of the velocity field at this plane indicates the existence of a vortex and a point of zero velocity $(y=1 / 2)$. For the largest Hartmann number analyzed $(\mathrm{Ha}=50)$, the behavior indicates that the magnetic field has an effect of suppressing convective currents inside the cavity. Results for the temperature field obtained by GITT are also compared with those of [82] in Fig. 5.b, in the horizontal midplane of the cavity $(y=1 / 2)$. Heat transfer by conduction clearly predominates at the largest Hartmann number $(\mathrm{Ha}=50)$. A marked change in temperature gradients is observed and the more significant presence of convection is observed as $\mathrm{Ha}$ is decreased towards the lowest Hartmann number $(\mathrm{Ha}=0)$, when magnetic effects cease. Furthermore, Figs. 5.a,b confirm the excellent agreement, to the graph scale, between the present GITT results with the numerical solution in [82].

The second Grashof number examined is much larger, $\mathrm{Gr}=10^{6}$, which corresponds to conditions in which thermal effects are of greater magnitude, with convection evolving much more rapidly and observing the emergence of marked movements of internal waves. Thus, the magnetic field necessary to suppress the natural convection must be stronger than previously considered for $\mathrm{Gr}=$ $10^{4}$. This behavior is shown in Figs. 6 for the streamfunction isolines with $\mathrm{Gr}=10^{6}$ and $\mathrm{Ha}=0$ and 100 at four different times. Now, with the presence of a stronger magnetic field, $\mathrm{Ha}=100$, with the advancement of the transient process, the axis of the central vortex is rotated in the counterclockwise direction, and this effect is due to the suppression of convection by the Lorentz force. Figures 7 show the isotherms for the cases $\mathrm{Gr}=10^{6}$ and $\mathrm{Ha}=0$ and 100 at four different times. Again, it is evident the formation of a distinct vertical boundary layer along the heated wall early in the process and, in the case of $\mathrm{Ha}=0$, the formation of at least two vortices at the geometric center of the cavity for the largest time, $t=0.93$. The discharge of a jet by the heated side wall forms an initially horizontal layer of intrusion that occurs along the upper horizontal wall of the cavity, as shown in Fig. 7.a for $t=0.005$. With the advancement of the transient process, the horizontal flow reaches the center of the cavity resulting in the formation of a thermally stratified core, where the temperature increases monotonically as a function of the coordinate $y$. 


\subsection{Primitive Variables Formulation: Transient lid driven cavity flow}

Figure 8.a shows a comparison of the horizontal velocity component for partial and total transformation for steady-state $(t=12)$, at the cavity centerline $(x=0.5)$, for uniform motion of the top lid, against results found in the literature, with overall good agreement to the graphical scale. Classical benchmark results were here adopted, including the works of Burggraf [88] and Ghia et al. [89], besides the error controlled GITT results in [25] and the more recent numerical simulation results in [90]. Figure 8.b shows a comparison of the vertical velocity component for partial and total transformation for stationary regime $(\mathrm{t}=12)$, at the cavity horizontal centerline $(\mathrm{y}=0.5)$, for uniform motion of the top lid, i.e., $\omega=0$.

Figure 9.a shows a comparison of the horizontal velocity component at the centreline of the cavity with oscillating lid velocity, at a specific instant of time for $\operatorname{Re}=100$ and $\omega=2 \pi / 6$, which agrees well with the numerical solution of Mendu and Das [86]. One may also note that the magnitude and direction of the velocity undergo a number of changes from the bottom to the top of the cavity. The centerline velocity profile in Fig. 9.a exhibits two local extremes due to the oscillatory motion. These repeated changes in magnitude and direction of the velocity along the centerline represent the presence of a number of complex vortices in the cavity. Fig. 9.b shows a comparison for the vertical velocity component at the horizontal centerline profile for six specific instants of time for $\operatorname{Re}=100$ and $=2 / 6$, where again one can observe a very good agreement with the results of Mendu and Das [86].

\subsection{Vectorial Eigenfunction Expansion: Membraneless redox flow batteries}

An application involving the mass transport of species in membraneless redox flow batteries $(\mathrm{MRFB})$ is here reviewed. Composed of two electrodes in contact with a flowing aqueous solution of ions of different electrochemical potentials, this device takes advantage of the laminar flow developed in microchannels to keep the two species separated without the need of a costly and often non-optimized ion selective membrane [91,92], but still avoiding each chemical substance to reach an undesirable electrode on the opposite side. To ensure good performance from the MRFB, a careful analysis of the mass transport must be carried out for both the separation of ions from the 
opposite electrode and for the effective delivery of ions to the reactive sites within the porous electrodes.

Figure 10 displays a model of the MRFB analyzed in the present work, consisting of symmetrically disposed porous carbon electrodes in flow-by configuration. Table 3 shows the convergence behavior of the eigenfunction expansion of Eq. (23) and a numerical comparison with the results obtained with the commercial software COMSOL Multiphysics. Convergence to at least three significant digits is observed with a maximum deviation from the finite elements results of $\sim 0.1 \%$. Figure 11.a depicts concentration contours to a typical electrochemical species with Schmidt number equal to 200. For $\operatorname{Re}=50$, full separation of the ions from the opposite electrode is obtained, which indicates the suitability of these operational parameters for a good performance of the MRFB. Figure 11.b shows the horizontal component of the velocity vector along the vertical direction, including the transition between fluid and porous layers. Results obtained with $C O M S O L$ Multiphysics are offered in a co-verification effort. The agreement is shown to be perfect to the graph scale, hence demonstrating the capability of the method described in section 4 of dealing with flow problems in heterogeneous media. Further calculations can be performed, aiming at determining the current density under mass-transport-limited conditions, for which the reader is referred to [61].

\section{CONCLUSIONS}

The Generalized Integral Transform Technique (GITT) is reviewed as a computationalanalytical approach in the analysis of transport phenomena based on formulations that involve the Navier-Stokes equations for the fluid flow modelling. Three alternative integral transform solution paths are revisited. Initially, the more commonly employed streamfunction-only formulation for two-dimensional flows is illustrated, which automatically satisfies the continuity equation and eliminates the pressure field. The first example deals with the streamfunction formulation in steadystate, when the eigenfunction expansion is obtained from a one-dimensional biharmonic eigenvalue problem, while the more challenging second example handles a transient situation, when the eigenvalue problem is two-dimensional for a total integral transformation scheme. Then, a primitive variables formulation is considered for both two and three-dimensional transient flows, which manipulates the momentum equations also to eliminate the pressure field, but employs the integral transformed continuity equation to provide one of the transformed velocity components in terms of the others. Finally, a recently introduced vector eigenfunction expansion strategy is discussed, as 
applied to both steady and transient two- or three-dimensional flows. As in the streamfunction formulation, this approach automatically satisfies continuity and eliminates the pressure field, recovering the streamfunction eigenfunction expansion for the two-dimensional case. This approach is also combined with a single domain reformulation strategy which allows for the straightforward treatment of heat (or mass) transfer and fluid flow in heterogeneous media. The approaches are demonstrated through examples involving laminar flow in annular channel with inner wall rotation, transient natural convection in magnetohydrodynamic flow, transient lid driven cavity flow problem, and mass transfer in membraneless redox flow batteries. These approaches should now be challenged in applications involving multiphase flow, flow instabilities, and complex fluids, to name a few new possibilities for extension of the hybrid numerical-analytical methodology.

\section{Acknowledgements}

The authors are grateful for the financial support offered by the Brazilian Government agencies CNPq (projects no. 401237/2014-1 and no. 207750/2015-7), CAPES-INMETRO, and FAPERJ. RMC is also grateful to the Leverhulme Trust for the Visiting Professorship (VP1-2017-028) at UCL and to the kind hospitality of the Department of Mechanical Engineering, University College London, UK.

This review paper is an extended version of the Plenary Lecture presented at the 10th Minsk International Seminar "Heat Pipes, Heat Pumps, Refrigerators, Power Sources", Minsk, Belarus, September 10th-13th, 2018, in celebration of the 50th anniversary of the foundation of the International Center for Heat and Mass Transfer, ICHMT.

\section{References}

1. Deakin M.A.B. Euler's invention of the integral transforms, Arch. Hist. Exact Sci. 1985, Vol.33, pp.307-319.

2. Fourier J.B. Théorie Analytique de la Chaleur, 1822, Firmin Didot Père et Fils., Paris. http://books.google.com/books?id=TDQJAAAAIAAJ.

3. Bogolyubov N.N., Faddeev L.D., Ishlinskii A.Yu., Koshlyakov V.N., Mitropol'skii Yu.A. Nikolai Sergeevich Koshlyakov (On the centenary of his birth). Russian Math. Surveys 1990, Vol.45, no.4, pp.197-202. 
4. Koshlyakov N.S. Basic Differential Equations of Mathematical Physics. (In Russian, “Osnovnye differentsial'nye itravneniya matematicheskii fiziki”), ONTI, Moscow, $4^{\text {th }}$ ed., 1936.

5. Koshlyakov N.S., Smirnov M.N., Gliner E.B. Differential Equations of Mathematical Physics. North Holland Publ. Co., Amsterdam, 1964 (transl. from Russian original 1951).

6. Eringen G.A. The Finite Sturm Liouville Transform. Technical report no.4, Office of Naval Research, USA, August 1953.

7. Luikov A.V. Analytical Heat Diffusion Theory, Academic Press, New York, 1968 (translation of 2nd ed. of Russian version published in 1967).

8. Mikhailov M.D. Nonstationary Temperature Fields in Skin, Energiya, Moscow, 1967.

9. Ozisik M.N. Boundary Value Problems of Heat Conduction, Int. Textbooks Co, 1968.

10. Mikhailov M.D., Özisik M.N. Unified Analysis and Solutions of Heat and Mass Diffusion, John Wiley, New York, 1984; also, Dover Publications, 1994.

11. Ozisik M.N., Murray R.L. On the Solution of Linear Diffusion Problems with Variable Boundary Condition Parameters, J. Heat Transfer, 1974, Vol. 96c, pp.48-51.

12. Mikhailov M.D. On the Solution of the Heat Equation with Time Dependent Coefficient, Int. J. Heat \& Mass Transfer, 1975, Vol.18, pp.344-345.

13. Cotta R.M. Diffusion in Media with Prescribed Moving Boundaries: Application to Metals Oxidation at High Temperatures, Proc. of the II Latin American Congress of Heat \& Mass Transfer, Vol. 1, pp. 502-513, São Paulo, Brasil, May 1986.

14. Cotta R.M., Ozisik M.N. Diffusion Problems with General Time-Dependent Coefficients, Rev. Bras. Ciências Mecânicas, 1987, Vol. 9, no. 4, pp. 269-292.

15. Cotta R.M., Santos C.A.C. Transient Diffusion Problems with Time-Dependent Boundary Condition Coefficients, J. Eng. Physics, 1992, Vol. 61, no. 5, pp. 1411-1418; also, in Russian, pp. 829-837, November 1991.

16. Cotta R.M. Hybrid Numerical-Analytical Approach to Nonlinear Diffusion Problems, Num. Heat Transfer, Part B, 1990, Vol. 17, pp. 217-226.

17. Serfaty R., Cotta R.M. Integral Transform Solutions of Diffusion Problems with Nonlinear Equation Coefficients, Int. Comm. Heat \& Mass Transfer, 1990, Vol. 17, no. 6, pp. 851-864.

18. Serfaty R., Cotta R.M. Hybrid Analysis of Transient Nonlinear Convection-Diffusion Problems, Int. J. Num. Meth. Heat \& Fluid Flow, 1992, Vol. 2, pp. 55-62. 
19. Leiroz A.J.K., Cotta R.M., On the Solution of Nonlinear Elliptic Convection-Diffusion Problems Through the Integral Transform Method, Num. Heat Transfer, Part B, 1993, Vol. 23, pp. 401-411.

20. Aparecido J.B., Cotta R.M., Ozisik M.N. Analytical Solutions to Two-Dimensional Diffusion Type Problems in Irregular Geometries, J. of the Franklin Institute, 1989, Vol. 326, pp. 421434.

21. Aparecido J.B., Cotta R.M. Laminar Flow Inside Hexagonal Ducts, Computational Mechanics, 1990, Vol. 6, pp. 93-100.

22. Aparecido J.B., Cotta R.M. Laminar Thermally Developing Flow Inside Right Triangular Ducts, Appl. Scientific Research, 1992, Vol. 49, pp. 355-368.

23. Sphaier L.A., Cotta R.M. Integral Transform Analysis of Multidimensional Eigenvalue Problems Within Irregular Domains, Num. Heat Transfer, Part B, 2000, Vol.38, pp.157-175.

24. Cotta R.M., Carvalho T.M.B. Hybrid Analysis of Boundary Layer Equations for Internal Flow Problems, 7th Int. Conf. on Num. Meth. in Laminar \& Turbulent Flow, Part 1, pp. 106-115, Stanford CA, July 1991.

25. Perez Guerrero J.S., Cotta R.M. Integral Transform Solution for the Lid-driven Cavity Flow Problem in Streamfunction-only Formulation, Int. J. Num. Meth. in Fluids, 1992, Vol. 15, pp. $399-409$.

26. Cotta R.M. Integral Transforms in Computational Heat and Fluid Flow. CRC Press, Boca Raton, FL, USA, 1993.

27. Cotta R.M. Benchmark Results in Computational Heat and Fluid Flow: -The Integral Transform Method, Int. J. Heat and Mass Transfer, 1994, Invited Paper, Vol. 37, pp.381-394.

28. Cotta R.M., Mikhailov M.D. Heat Conduction: Lumped Analysis, Integral Transforms, Symbolic Computation, Wiley-Interscience, New York, 1997.

29. Cotta R.M. The Integral Transform Method in Thermal \& Fluids Sciences \& Engineering, Begell House, New York, USA, 1998.

30. Cotta R.M., Mikhailov M.D. Hybrid Methods and Symbolic Computations. Handbook of Numerical Heat Transfer, 2nd ed., Chap. 16, W.J. Minkowycz, E.M. Sparrow, and J.Y. Murthy, Eds., John Wiley, New York, 2006.

31. Cotta R.M., Knupp D.C., Naveira-Cotta C.P. Analytical Heat and Fluid Flow in Microchannels and Microsystems, Mechanical Eng. Series, Springer, New York, 2016. 
32. I. F. Pinheiro, L. A. Sphaier \& L. S. de B. Alves (2018) Integral transform solution of integrodifferential equations in conduction-radiation problems, Numerical Heat Transfer, Part A: Applications, 2018, Vol.73, no.2, pp.94-114.

33. Cotta R.M., Knupp D.C., Quaresma J.N.N. Analytical Methods in Heat Transfer, In: Handbook of Thermal Science and Engineering, F.A. Kulacki et al., Eds., Springer, 2018.

34. Cotta R.M., Naveira-Cotta C.P., Knupp D.C., Zotin J.L.Z., Pontes P.C., Almeida A.P. Recent Advances in Computational-Analytical Integral Transforms for Convection-Diffusion Problems, Heat \& Mass Transfer, Invited Paper, 2018, Vol.54, pp.2475-2496.

35. Perez Guerrero J.S., Cotta R.M. Integral Transform Solution of Developing Laminar Duct Flow in Navier-Stokes Formulation, Int. J. Num. Meth. in Fluids, 1995, Vol. 20, pp. 1203-1213.

36. Perez Guerrero J.S., Cotta R.M. Benchmark Integral Transform Results for Flow Over a Backward - Facing Step, Computers \& Fluids, 1996, Vol.25, no.5, pp.527-540.

37. Lima J.A., Perez Guerrero J.S., Cotta R.M. Hybrid Solution of the Averaged Navier-Stokes Equations for Turbulent Flow, Computational Mechanics, 1997, Vol.19, no.4, pp.297-307.

38. Pereira L.M., Perez Guerrero J.S., Cotta R.M. Integral Transformation of the Navier-Stokes Equations in Cylindrical Geometry, Computational Mechanics, 1998, Vol.21, no.1, pp.60-70.

39. Leal M.A., Perez Guerrero J.S., Cotta R.M. Natural Convection Inside Two-Dimensional Cavities: The Integral Transform Method, Comm. Num. Meth. Eng., 1999, Vol.15, pp.113-125.

40. Perez Guerrero J.S., Quaresma J.N.N., Cotta R.M. Simulation of Laminar Flow Inside Ducts of Irregular Geometry Using Integral Transforms, Computational Mechanics, 2000, Vol.25, no.4, pp.413-420.

41. Leal M.A., Machado H.A., Cotta R.M. Integral Transform Solutions of Transient Natural Convection in Enclosures with Variable Fluid Properties, Int. J. Heat \& Mass Transfer, 2000, Vol.43, no.21, pp.3977-3990.

42. Pereira L.M., Cotta R.M., Perez Guerrero J.S. Analysis of Laminar Forced Convection in Annular Ducts Using Integral Transforms, Hybrid Meth. Eng., 2000, Vol.2, no.2, pp.221-232.

43. Ramos R., Perez Guerrero J.S., Cotta R.M. Stratified Flow Over a Backward Facing Step:Hybrid Solution by Integral Transforms, Int. J. Num. Meth. in Fluids, 2001, Vol.35, no.2, pp.173-197.

44. Silva C. A. M., Macedo E.N., Quaresma J.N.N., Pereira L.M., Cotta R.M. Integral Transform Solution of the Navier-Stokes Equations in Full Cylindrical Regions with Streamfunction Formulation, Int. J. Num. Meth. Biomedical Eng., 2010, Vol.26, pp.1417-1434. 
45. Matt C.F.T., Quaresma J.N.N., Cotta R.M. Analysis of Magnetohydrodynamic Natural Convection in Closed Cavities through Integral Transforms, Int. J. Heat \& Mass Transfer, 2017, Vol.113, pp.502-513.

46. Pontes F.A., Macedo E.N., Batista C.S., Lima J.A., and Quaresma J.N.N. Hybrid Solutions Obtained via Integral Transforms for Magnetohydrodynamic Flow with Heat Transfer in Parallel Plate Channels, Int. J. Num. Meth. Heat Fluid Flow, 2018, Vol.28, pp.1474-1505.

47. Lima G.G.C., Santos C.A.C., Haag A., Cotta R.M. Integral Transform Solution of Internal Flow Problems Based on Navier-Stokes Equations and Primitive Variables Formulation, Int. J. Num. Meth. Eng., 2007, Vol.69, pp.544-561.

48. Curi M.F., Cotta R.M., Perez-Guerrero J.S. Alternative Integral Transforms Solution of the Transient Incompressible Navier-Stokes Equations in Primitive Variables Formulation, ENCIT 2014, 15th Brazilian Congress of Thermal Sciences and Engineering, November 10-13, 2014, Belém, PA, Brazil.

49. Quaresma J.N.N., Cotta R.M. Integral Transform Method for the Navier-Stokes Equations in Steady Three-Dimensional Flow, Proc. of the 10th ISTP - Int. Symp. on Transport Phenomena, pp.281-287, Kyoto, Japan, November, 1997.

50. Cotta R.M., Mikhailov M.D. The Integral Transform Method, Appl. Math. Modelling, 1993, Vol. 17, pp. 156-161.

51. Carvalho T.M.B., Cotta R.M., Mikhailov M.D. Flow Development in the Entrance Regions of Ducts, Comm. Num. Meth. Eng., 1993, Vol.9, pp. 503-509.

52. Machado H.A., Cotta R.M. Integral Transform Method for Boundary Layer Equations in Simultaneous Heat and Fluid Flow Problems, Int. J. Num. Meth. Heat \& Fluid Flow, 1995, Vol. 5, pp. 225-237.

53. Bolivar M.A.H., Lage P.L.C., Cotta R.M. Integral Transform Solution of the Laminar Thermal Boundary Layer Problem for Flow Past Two-Dimensional and Axisymmetric Bodies, Num. Heat Transfer, part A, 1998, Vol.33, no.7, pp.779-797.

54. Cotta R.M., Pimentel L.C.G., Developing Turbulent Duct Flow: - Hybrid Solution Via Integral Transforms and Algebraic Models, Int. J. Num. Meth. in Heat \& Fluid Flow, 1998, Vol.8, no.1, pp.10-26.

55. Knupp D.C., Cotta R.M., Naveira-Cotta C.P., Heat transfer in microchannels with upstreamdownstream regions coupling and wall conjugation effects, Numer. Heat Transfer, Part B, 2013, Vol. 64, pp. 365-387. 
56. Knupp D.C., Naveira-Cotta C.P., Cotta R.M., Theoretical-experimental analysis of conjugated heat transfer in nanocomposite heat spreaders with multiple microchannels, Int. J. Heat Mass Transf., 2014, Vol. 74, pp. 306-318.

57. Knupp, D.C. Cotta R.M., Naveira-Cotta C.P., Kakaç S. Transient conjugated heat transfer in microchannels: Integral transforms with single domain formulation, Int. J. Therm. Sci., 2015, Vol. 88, pp. 248-257.

58. Knupp D.C., Cotta R.M., Naveira-Cotta, C.P. Fluid flow and conjugated heat transfer in arbitrarily shaped channels via single domain formulation and integral transforms, Int. J. Heat Mass Transf., 2015, Vol. 82, pp. 479-489.

59. Almeida A.P., Naveira-Cotta C.P., Cotta R.M. Integral Transforms for Transient Threedimensional Heat Conduction in Heterogeneous Media with Multiple Geometries and Materials, Paper \# IHTC16-24583, Proc. of the 16th International Heat Transfer Conference - IHTC16, 2018, Beijing, China, August 10th-15th.

60. Lisboa K.M., Cotta R.M., Hybrid Integral Transforms for Flow Development in Ducts Partially Filled with Porous Media, Proc. Royal Society A - Mathematical, Physical and Eng. Sciences, 2018, Vol. 474, pp.1-20.

61. Lisboa K.M., Cotta R.M., On the Mass Transport in Membraneless Flow Batteries of Flow-by Configuration, Int. J. Heat \& Mass Transfer, 2018, Vol.122, pp.954-966.

62. Cotta R.M., Su J., Pontedeiro A.C., Lisboa K.M. Computational-Analytical Integral Transforms and Lumped-Differential Formulations: Benchmarks and Applications in Nuclear Technology, Invited Plenary Lecture, Int. Symp. on Turbulence, Heat and Mass Transfer, THMT-ICHMT, Begell House, Rio de Janeiro, July 10th-13th, 2018.

63. Lisboa K.M., Su J., Cotta R.M., Single Domain Integral Transforms Analysis of Natural Convection in Cavities Partially Filled with Heat Generating Porous Medium, Num. Heat Transfer, Part A, 2018, Vol.74, no.3, pp.1068-1086.

64. Hirata S.C., Goyeau B., Gobin D., Cotta R.M. Stability of Natural Convection in Composite Enclosures using Integral Transforms, Num. Heat Transfer, Part B, 2006, Vol.50, pp.409-424.

65. Hirata S.C., Goyeau B., Gobin D., Carr M., Cotta R.M., Linear stability of natural convection in superposed fluid and porous layers: Influence of the interfacial modelling, Int. J. Heat Mass Transf., 2007, Vol. 50, no.7-8, pp.1356-1367. 
66. Hirata S.C., Goyeau B., Gobin D., Chandesris, Jamet D., Stability of natural convection in superposed fluid and porous layers: Equivalence of the one- and two-domain approaches, Int. J. Heat Mass Transf., 2009, Vol. 52, pp. 533-536, 2009.

67. Alves L.S.B., Cotta R.M., Transient Natural Convection Inside Porous Cavities: Hybrid Numerical-Analytical Solution and Mixed Symbolic-Numerical Computation, Num. Heat Transfer, Part A, 2000, Vol. 38, no.1, pp.89-110.

68. Luz Neto H., Quaresma J.N.N., Cotta R.M., Natural Convection in Three-Dimensional Porous Cavities: Integral Transform Method, Int. J. Heat \& Mass Transfer, 2002, Vol.45, no.14, pp.3013-3032.

69. Alves L.S.B., Cotta R.M., Pontes J., Stability Analysis of Natural Convection in Porous Cavities Through Integral Transforms, Int. J. Heat \& Mass Transfer, 2002, Vol.45, pp.1185-1195.

70. Luz Neto H., Quaresma J.N.N., Cotta R.M., Integral Transform Solution for Transient Natural Convection in Three-Dimensional Porous Cavities: Aspect Ratio Effects, Int. J. Heat and Mass Transfer, 2006, Vol.49, pp.4687-4695.

71. Cotta R.M., Knupp D.C., Naveira-Cotta C.P., Sphaier L., Quaresma J.N.N., Unified Integral Transforms Algorithm for Solving Multidimensional Nonlinear Convection-Diffusion Problems, Num. Heat Transfer-Part A, 2013, Vol.63, pp.1-27.

72. Cotta R.M., Knupp D.C., Naveira-Cotta C.P., Sphaier L., Quaresma J.N.N., The Unified Integral Transforms (UNIT) Algorithm with Total and Partial Transformation, Comput. Thermal Sciences, 2014, Vol.6, no.6, pp.507-524.

73. Cotta R.M., Naveira-Cotta C.P., Knupp D.C. Convective Eigenvalue Problems for Convergence Enhancement of Eigenfunction Expansions in Convection-Diffusion Problems, ASME J. Thermal Science and Eng. Appl., 2017, Vol.10, no.2, pp.021009 (12 pages).

74. Cotta R.M., Naveira-Cotta C.P., Knupp D.C. Nonlinear Eigenvalue Problem in the Integral Transforms Solution of Convection-Diffusion with Nonlinear Boundary Conditions, Int. J. Num. Meth. Heat \& Fluid Flow, 2016, Invited Paper, 25th Anniversary Special Issue, Vol.26, nos.3\&4, pp.767-789.

75. Cotta R.M., Naveira-Cotta C.P., Knupp D.C. Enhanced Eigenfunction Expansions in Convection-Diffusion Problems with Multiscale Space Variable Coefficients, Num. Heat Transfer, part A - Applications, 2016, Vol.70, no.5, pp.492-512. 
76. Cotta R.M., Gerk J.E.V. Mixed Finite Difference/Integral Transform Approach for ParabolicHyperbolic Problems in Transient Forced Convection, Numerical Heat Transfer - Part B: Fundamentals, 1994, Vol. 25, pp. 433-448.

77. Wolfram S. Mathematica, version 11, Wolfram Research Inc., Champaign, 2017.

78. Coney J.E.R., El-Shaarawi M.A.I. A Contribution to the Numerical Solution of Developing Laminar Flow in the Entrance Region of Concentric Annuli with Rotating Inner Walls, ASME Journal of Fluids Engineering, 1974, Series I, no. 4, pp. 333-340.

79. Amorim N.S. Hybrid Solutions in the Analysis of Laminar Flow in Concentric Annular Ducts with Inner Wall Rotation. MSc dissertation (In Portuguese), Graduate Program in Chemical Engineering, Federal University of Pará, UFPA, Belém, Brazil, 2009.

80. IMSL Library, MATH/LIB, 1991, Houston, TX.

81. Silva J.J.G., Macedo E.N., Quaresma, J.N.N., Cotta R.M. Hybrid Integral Transform Solution for MHD Natural Convection in Cavities, 21st International Congress of Mechanical Engineering, COBEM-2011, ABCM, Natal, RN, Brazil, October 2011.

82. Colaço M.J., Dulikravich G.S., Orlande H.R.B. Magnetohydrodynamic Using Radial Basis Functions, Int. J. Heat Mass Transfer, 2009, Vol. 52, pp. 5932-5939.

83. Job V.M., Gunakala S.R. Unsteady MHD Free Convection Nanofluid Flows within a Wavy Trapezoidal Enclosure with Viscous and Joule Dissipation Effects, Numerical Heat Transfer, Part A: Applications, 2016, Vol.69, no.4, pp.421-443.

84. Son J.H., Park I.S. Numerical Study of MHD Natural Convection in a Rectangular Enclosure with an Insulated Block, Numerical Heat Transfer, Part A: Applications, 2017, Vol.71, no.10, pp.1004-1022.

85. Ahrar A.J., Djavareshkian M.H. Novel Hybrid Lattice Boltzmann Technique with TVD Characteristics for Simulation of Heat Transfer and Entropy Generations of MHD and Natural Convection in a Cavity, Numerical Heat Transfer, Part B: Fundamentals, 2017, Vol.72, no.6, pp.431-449.

86. Mendu S.S., Das P.K. Fluid Flow in a Cavity Driven by an Oscillating Lid - A Simulation By Lattice-Boltzmann Method, Eur. J. Mech. B/Fluids., 2013, Vol. 39, pp. 59-70.

87. Lisboa K.M., Su J., Cotta R.M. Vector Eigenfunction Expansion in the Integral Transform Solution of Transient Natural Convection, Int. Journal of Num. Meth. Heat and Fluid Flow, to appear. 
88. Burggraf O.D. Analytical and Numerical Studies of the Structure of Steady Separated Flows. J. Fluid Mech., 1966, Vol. 24, no. 1, pp. 113-151.

89. Ghia U., Ghia K.N., Shin C.T. High-Re Solutions for Incompressible Flow Using the NavierStokes Equations and a Multigrid Method, J. Comp. Phys., 1982, Vol.48, pp. 387-411.

90. Aydin M., Fenner R.T. Boundary Element Analysis of Driven Cavity Flow for Low and Moderate Reynolds Numbers, Int. J. Numer. Meth. Fluids, 2001, Vol.37, pp. 45-64.

91. Lee J.W., Goulet M.-A., Kjeang E. Microfluidic Redox Battery, Lab Chip, 2013, Vol. 13, pp. 2504.

92. Lisboa K.M., Marschewski J., Ebejer N., Ruch P., Cotta R.M., Michel B., Poulikakos D. Mass Transport Enhancement in Redox Flow Batteries with Corrugated Fluidic Networks, J. Power Sources, 2017, Vol. 359, pp. 322-331. 
Table 1 - Analysis of the convergence of the axial velocity componet, $v_{z}(r . z)$, for the case with rotation of the inner cylinder $(\xi=1)$ for $\operatorname{Re}=300$ and $\gamma=0.1$.

\begin{tabular}{|c|c|c|c|c|c|}
\hline \multirow{2}{*}{$\mathrm{N}$} & \multicolumn{5}{|c|}{$\mathrm{z}=0.54$} \\
\cline { 2 - 6 } & $\mathrm{r}=0.145$ & $\mathrm{r}=0.325$ & $\mathrm{r}=0.550$ & $\mathrm{r}=0.775$ & $\mathrm{r}=0.955$ \\
\hline 7 & 0.9130 & 1.092 & 1.090 & 1.186 & 0.5439 \\
\hline 11 & 0.6621 & 1.095 & 1.106 & 1.193 & 0.5049 \\
\hline 15 & 0.6117 & 1.106 & 1.109 & 1.201 & 0.4985 \\
\hline 19 & 0.6024 & 1.108 & 1.109 & 1.204 & 0.4966 \\
\hline 23 & 0.5993 & 1.109 & 1.109 & 1.205 & 0.4959 \\
\hline 27 & 0.5981 & 1.110 & 1.109 & 1.205 & 0.4956 \\
\hline 31 & 0.5976 & 1.110 & 1.109 & 1.206 & 0.4955 \\
\hline 35 & 0.5973 & 1.110 & 1.109 & 1.206 & 0.4955 \\
\hline 39 & 0.5972 & 1.110 & 1.109 & 1.206 & 0.4955 \\
\hline $\mathrm{N}$ & \multicolumn{5}{|l}{} \\
\hline 7 & $\mathrm{r}=0.145$ & $\mathrm{r}=0.325$ & $\mathrm{r}=0.550$ & $\mathrm{r}=0.775$ & $\mathrm{r}=0.955$ \\
\hline 11 & 0.5126 & 1.285 & 1.382 & 1.120 & 0.2891 \\
\hline 15 & 0.5041 & 1.095 & 1.106 & 1.193 & 0.2880 \\
\hline 19 & 0.5025 & 1.276 & 1.390 & 1.119 & 0.2877 \\
\hline 23 & 0.5021 & 1.275 & 1.390 & 1.120 & 0.2877 \\
\hline 27 & 0.5020 & 1.275 & 1.390 & 1.120 & 0.2877 \\
\hline 31 & 0.5020 & 1.275 & 1.390 & 1.120 & 0.2877 \\
\hline 35 & 0.5020 & 1.275 & 1.390 & 1.120 & 0.2877 \\
\hline 39 & 0.5019 & 1.275 & 1.390 & 1.120 & 0.2877 \\
\hline & 0.5019 & 1.275 & 1.390 & 1.120 & 0.2877 \\
\hline
\end{tabular}


Table 2 - Convergence behavior for the global mean Nusselt number, streamfunction modulus and temperature for $\mathrm{t}=0.005$ and in the steady state with $\mathrm{Gr}=10^{4}, \mathrm{Pr}=0.71$ and $\mathrm{Ha}=0$.

\begin{tabular}{|c|c|c|c|c|c|}
\hline \multicolumn{6}{|c|}{$\mathrm{t}=0.005$} \\
\hline $\mathrm{N}$ & $\overline{\mathrm{N}} \mathrm{u}$ & $(0.1,0.1)$ & $\mathrm{T}(0.1,0.1)$ & $(0.9,0.9)$ & $\mathrm{T}(0.9,0.9)$ \\
\hline 40 & 1.000 & $0.198 \times 10^{-2}$ & 0.309 & $0.100 \times 10^{-3}$ & 0 \\
\hline 80 & 1.001 & $0.257 \times 10^{-2}$ & 0.305 & $0.788 \times 10^{-4}$ & 0 \\
\hline 120 & 1.001 & $0.267 \times 10^{-2}$ & 0.304 & $0.540 \times 10^{-4}$ & 0 \\
\hline 160 & 1.001 & $0.267 \times 10^{-2}$ & 0.304 & $0.488 \times 10^{-4}$ & 0 \\
\hline 200 & 1.001 & $0.264 \times 10^{-2}$ & 0.304 & $0.515 \times 10^{-4}$ & 0 \\
\hline 240 & 1.001 & $0.263 \times 10^{-2}$ & 0.304 & $0.557 \times 10^{-4}$ & 0 \\
\hline 260 & 1.001 & $0.261 \times 10^{-2}$ & 0.303 & $0.501 \times 10^{-4}$ & 0 \\
\hline 280 & 1.001 & $0.261 \times 10^{-2}$ & 0.303 & $0.520 \times 10^{-4}$ & 0 \\
\hline 300 & 1.001 & $0.261 \times 10^{-2}$ & 0.304 & $0.541 \times 10^{-4}$ & 0 \\
\hline \multicolumn{6}{|c|}{ steady state } \\
\hline $\mathrm{N}$ & $\overline{\mathrm{Nu}}$ & $(0.1,0.1)$ & $\mathrm{T}(0.1,0.1)$ & $(0.9,0.9)$ & $\mathrm{T}(0.9,0.9)$ \\
\hline 40 & 2.009 & $0.321 \times 10^{-2}$ & 0.698 & $0.321 \times 10^{-2}$ & 0.301 \\
\hline 80 & 2.010 & $0.349 \times 10^{-2}$ & 0.696 & $0.349 \times 10^{-2}$ & 0.303 \\
\hline 120 & 2.010 & $0.353 \times 10^{-2}$ & 0.695 & $0.353 \times 10^{-2}$ & 0.304 \\
\hline 160 & 2.010 & $0.353 \times 10^{-2}$ & 0.695 & $0.353 \times 10^{-2}$ & 0.304 \\
\hline 200 & 2.010 & $0.352 \times 10^{-2}$ & 0.695 & $0.352 \times 10^{-2}$ & 0.304 \\
\hline 240 & 2.010 & $0.352 \times 10^{-2}$ & 0.695 & $0.352 \times 10^{-2}$ & 0.304 \\
\hline 260 & 2.010 & $0.352 \times 10^{-2}$ & 0.695 & $0.352 \times 10^{-2}$ & 0.304 \\
\hline 280 & 2.010 & $0.352 \times 10^{-2}$ & 0.695 & $0.352 \times 10^{-2}$ & 0.304 \\
\hline 300 & 2.010 & $0.352 \times 10^{-2}$ & 0.695 & $0.352 \times 10^{-2}$ & 0.304 \\
\hline
\end{tabular}


Table 3: Convergence behavior and co-verification of the GITT and COMSOL results for the horizontal velocity component at the channel centerline in selected longitudinal positions.

\begin{tabular}{|c|c|c|c|c|c|}
\hline \multicolumn{7}{|c|}{$\mathrm{Re}=50 ; \mathrm{Da}=0.002 ; \mathrm{y}=0$} \\
\hline & $\mathrm{x}=0.1$ & $\mathrm{x}=0.2$ & $\mathrm{x}=0.4$ & $\mathrm{x}=0.6$ & $\mathrm{x}=0.8$ \\
\hline $\mathrm{N}=3$ & 0.984 & 1.045 & 1.182 & 1.272 & 1.317 \\
\hline $\mathrm{N}=6$ & 1.009 & 1.065 & 1.187 & 1.273 & 1.317 \\
\hline $\mathrm{N}=9$ & 1.027 & 1.067 & 1.186 & 1.273 & 1.317 \\
\hline $\mathrm{N}=12$ & 1.018 & 1.066 & 1.186 & 1.273 & 1.317 \\
\hline $\mathrm{N}=15$ & 1.017 & 1.066 & 1.186 & 1.273 & 1.317 \\
\hline $\mathrm{N}=18$ & 1.019 & 1.066 & 1.187 & 1.273 & 1.317 \\
\hline $\mathrm{COMSOL}$ & 1.019 & 1.067 & 1.187 & 1.274 & 1.319 \\
\hline
\end{tabular}




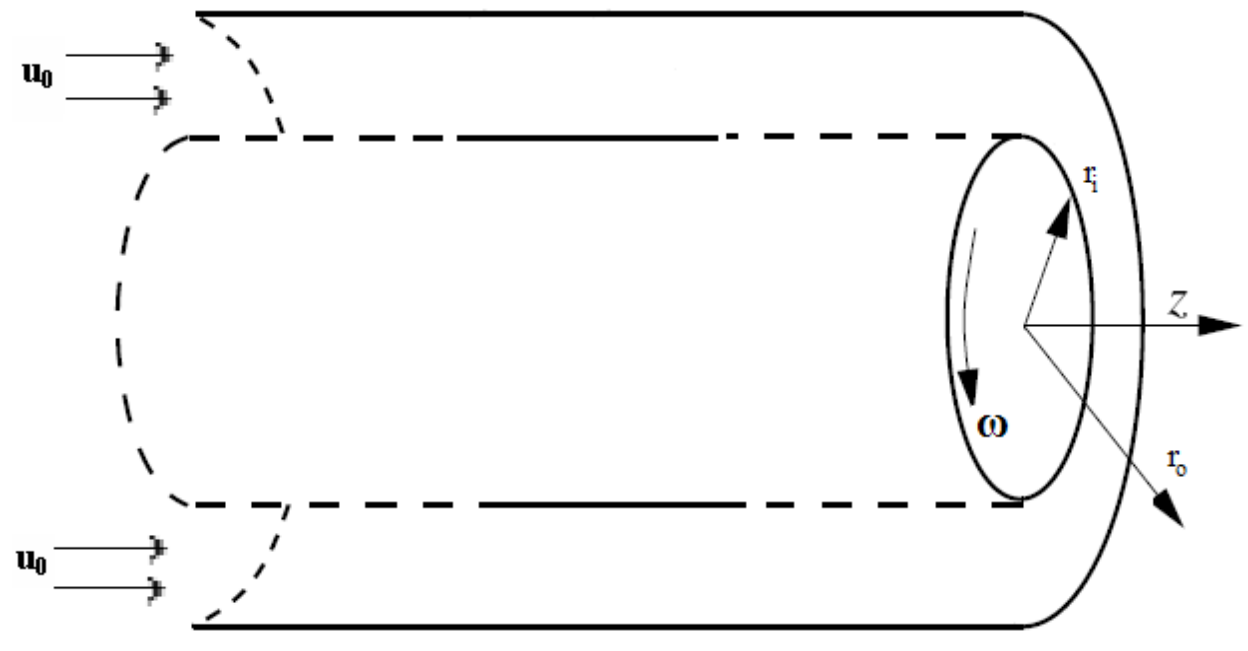

Figure 1 - Geometry and coordinates for laminar flow within annular duct with rotating inner wall. 


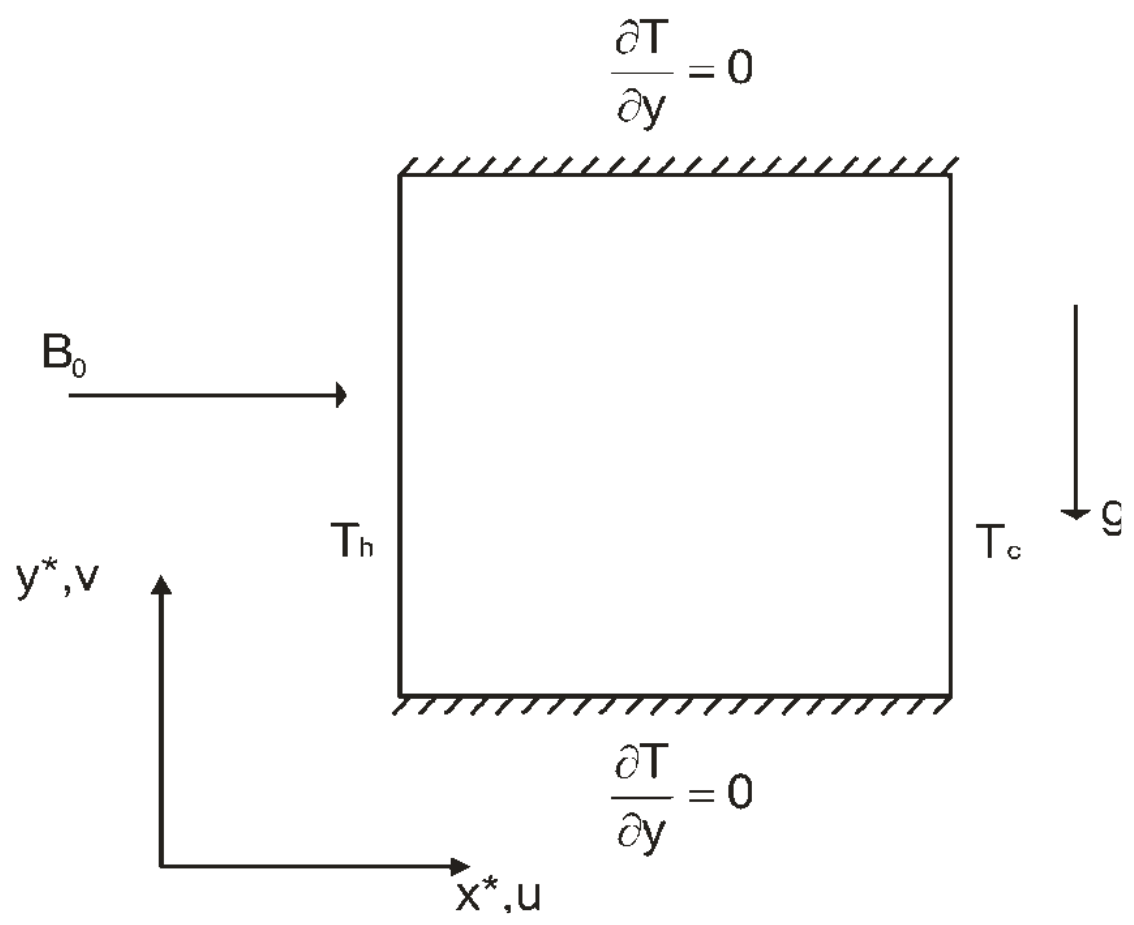

Figure 2- Geometry and coordinates system for natural convection in square cavity with a transversal magnetic field. 


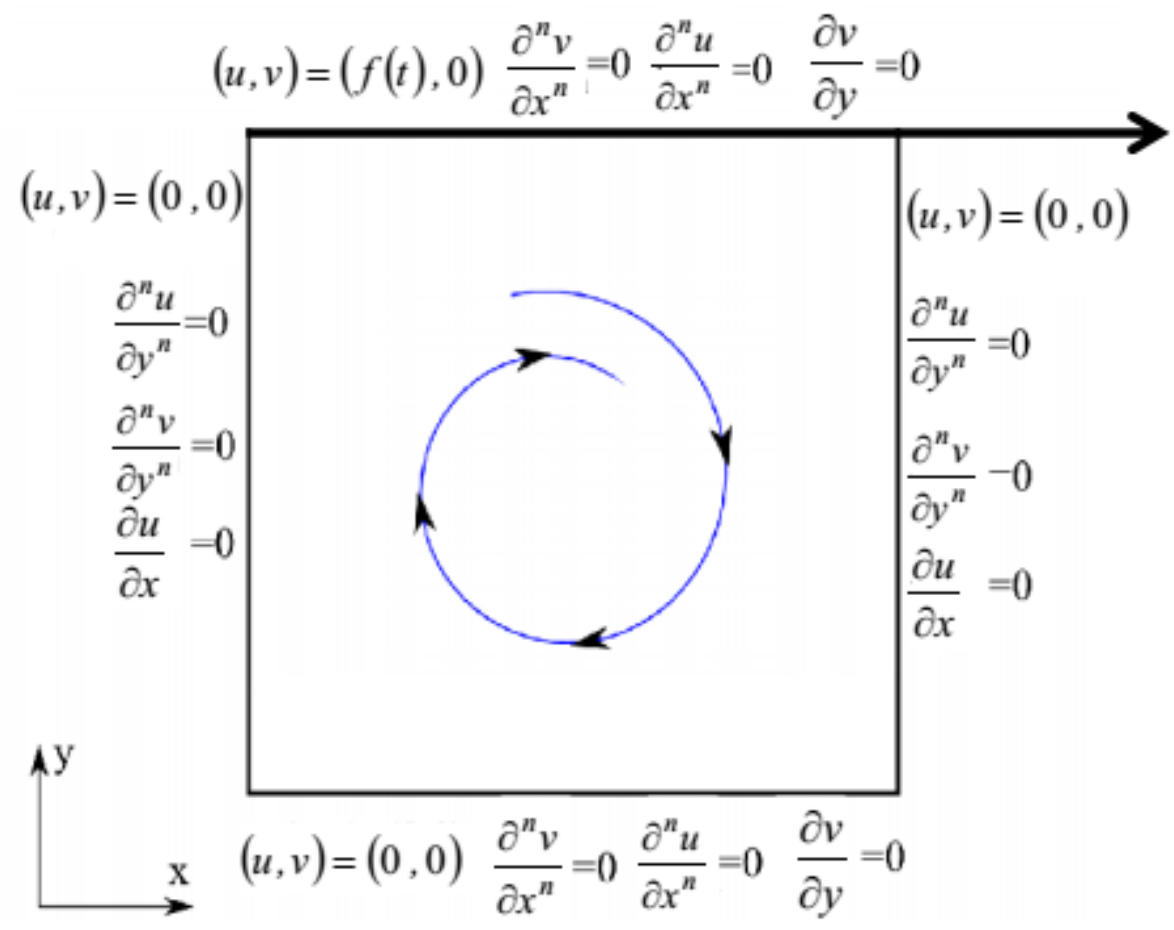

Figure 3: Schematic representation of the transient lid-driven cavity flow problem 


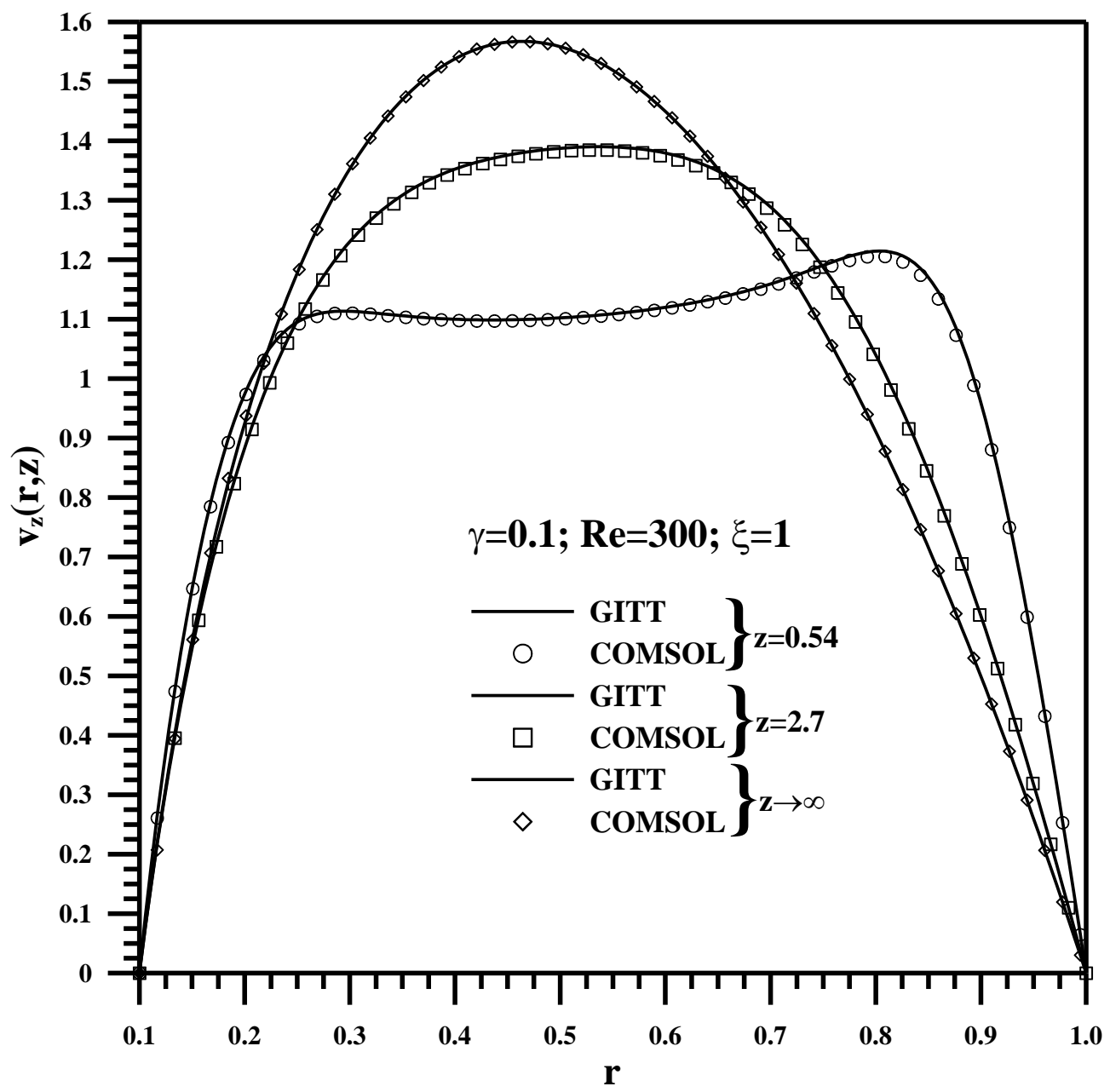

Figure 4 - Development of the axial velocity component $\mathrm{v}_{\mathrm{z}}(\mathrm{r}, \mathrm{z})$ for flow in annular channel with inner wall rotation $(\operatorname{Re}=300, \gamma=0.1$ and $\xi=1)$. 


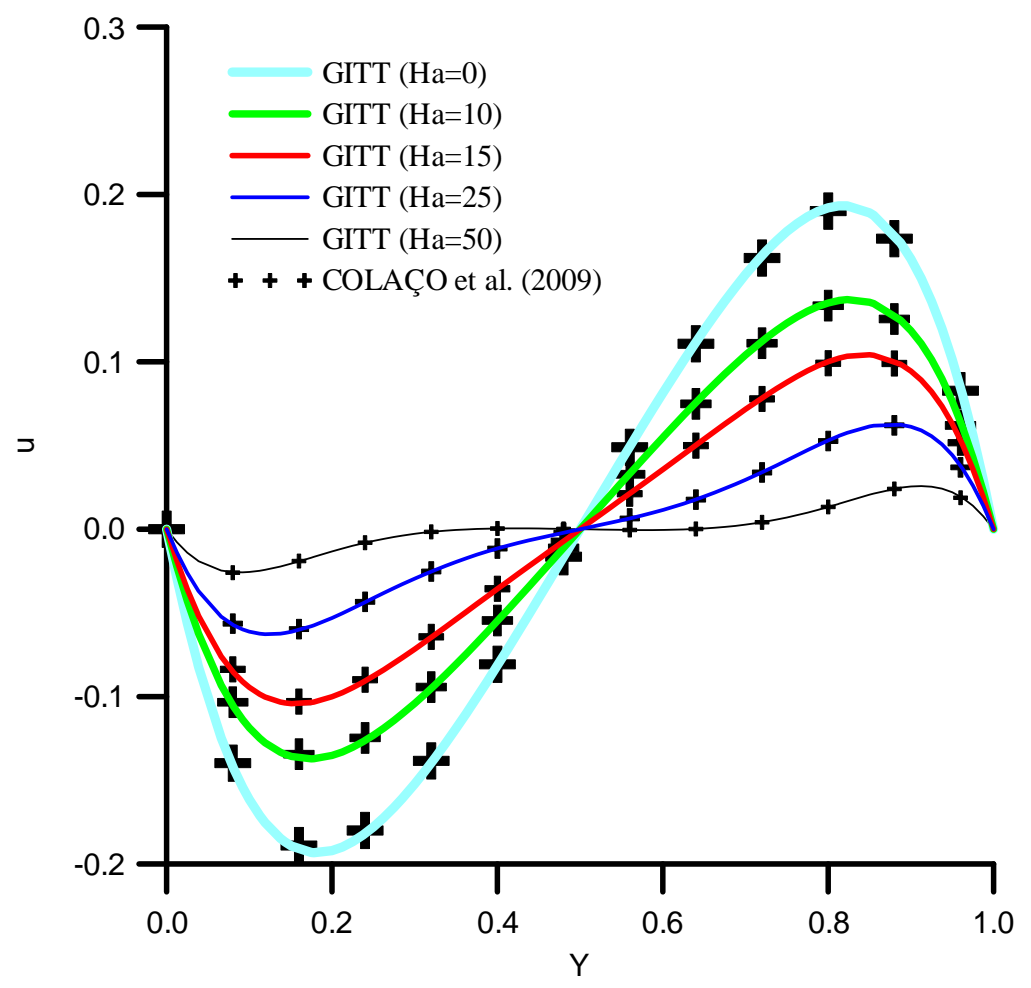

(a)

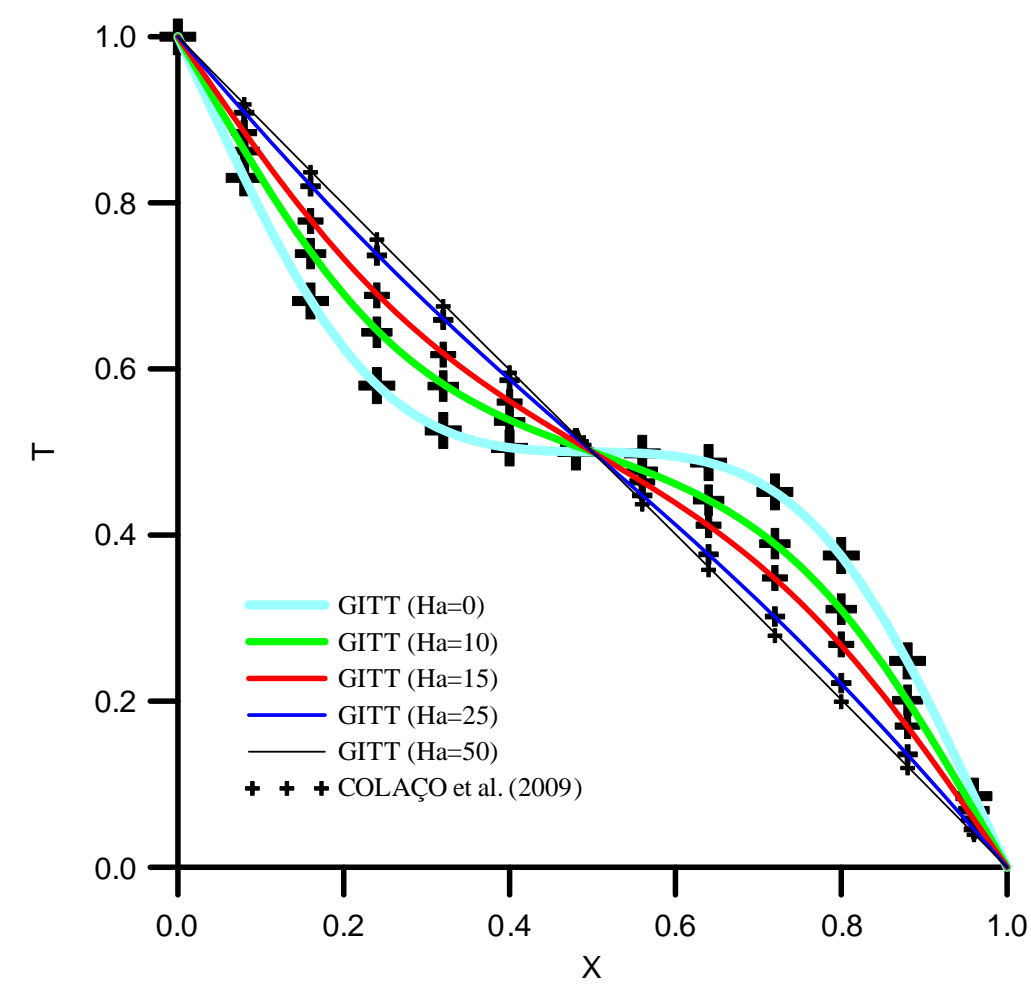

(b)

Figures 5. Comparison of the velocity $\mathrm{x}$-component and temperature fields for $\mathrm{Gr}=10^{4}$ at steady state: (a) velocity component $u$ along the vertical coordinate at the cavity plane $x=1 / 2$;

(b) temperature profile along the horizontal coordinate at the cavity plane $y=1 / 2$. 


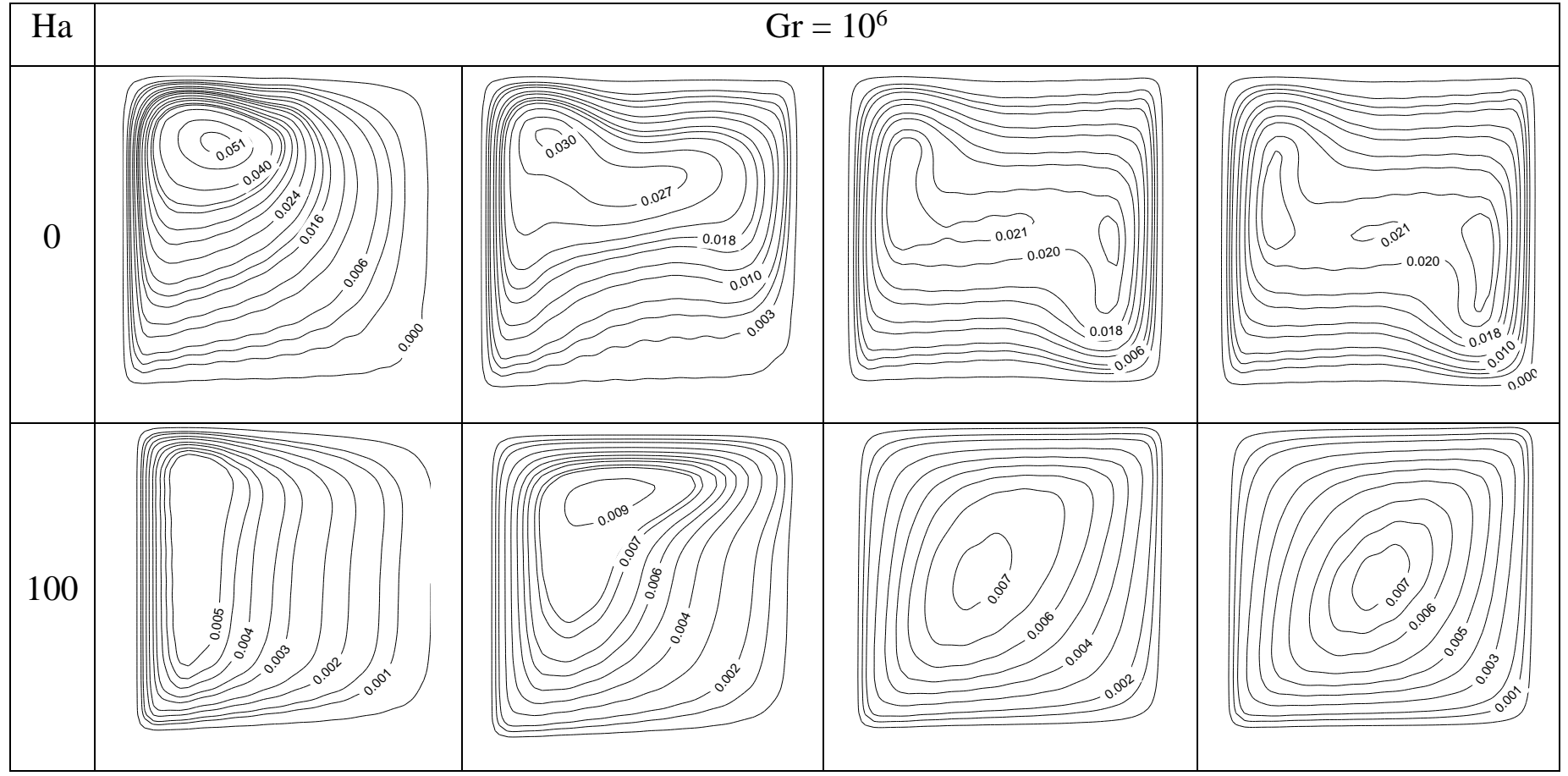

(a) (b) (d)

Figures 6. Transient streamfunction isolines at different times with $\mathrm{Gr}=10^{6}$ and $\mathrm{Ha}=0$ and 100.

$$
\text { (a) } \mathrm{t}=0.005 \text {; (b) } \mathrm{t}=0.02 \text {; (c) } \mathrm{t}=0.1 \text {; (d) } \mathrm{t}=0.93 \text {. }
$$




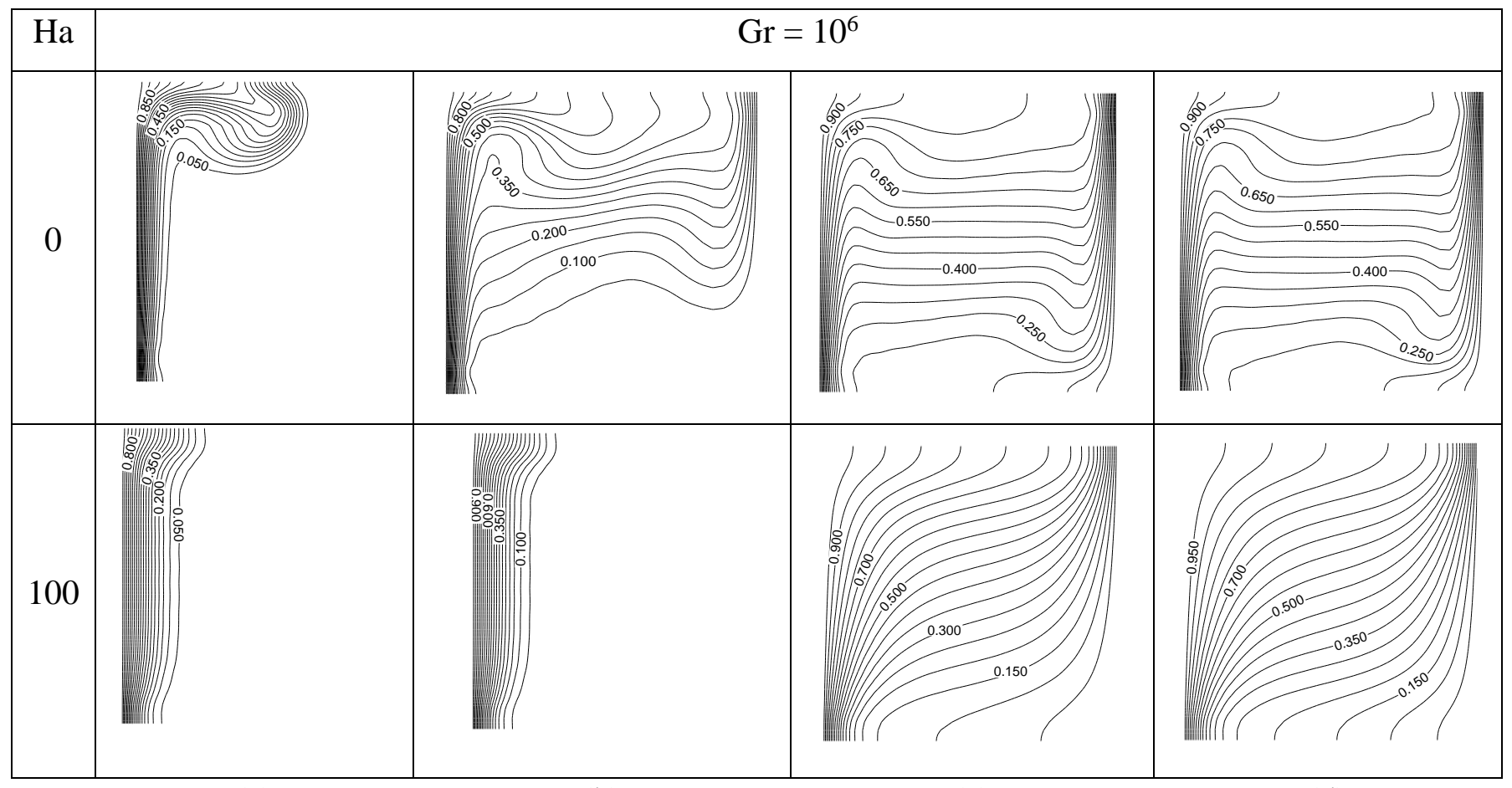

(a)

(b)

(c)

(d)

Figures 7. Transient temperature isolines at different times with $\mathrm{Gr}=10^{6}$ and $\mathrm{Ha}=0$ and 100.

(a) $\mathrm{t}=0.005$; (b) $\mathrm{t}=0.02$; (c) $\mathrm{t}=0.1$; (d) $\mathrm{t}=0.93$.

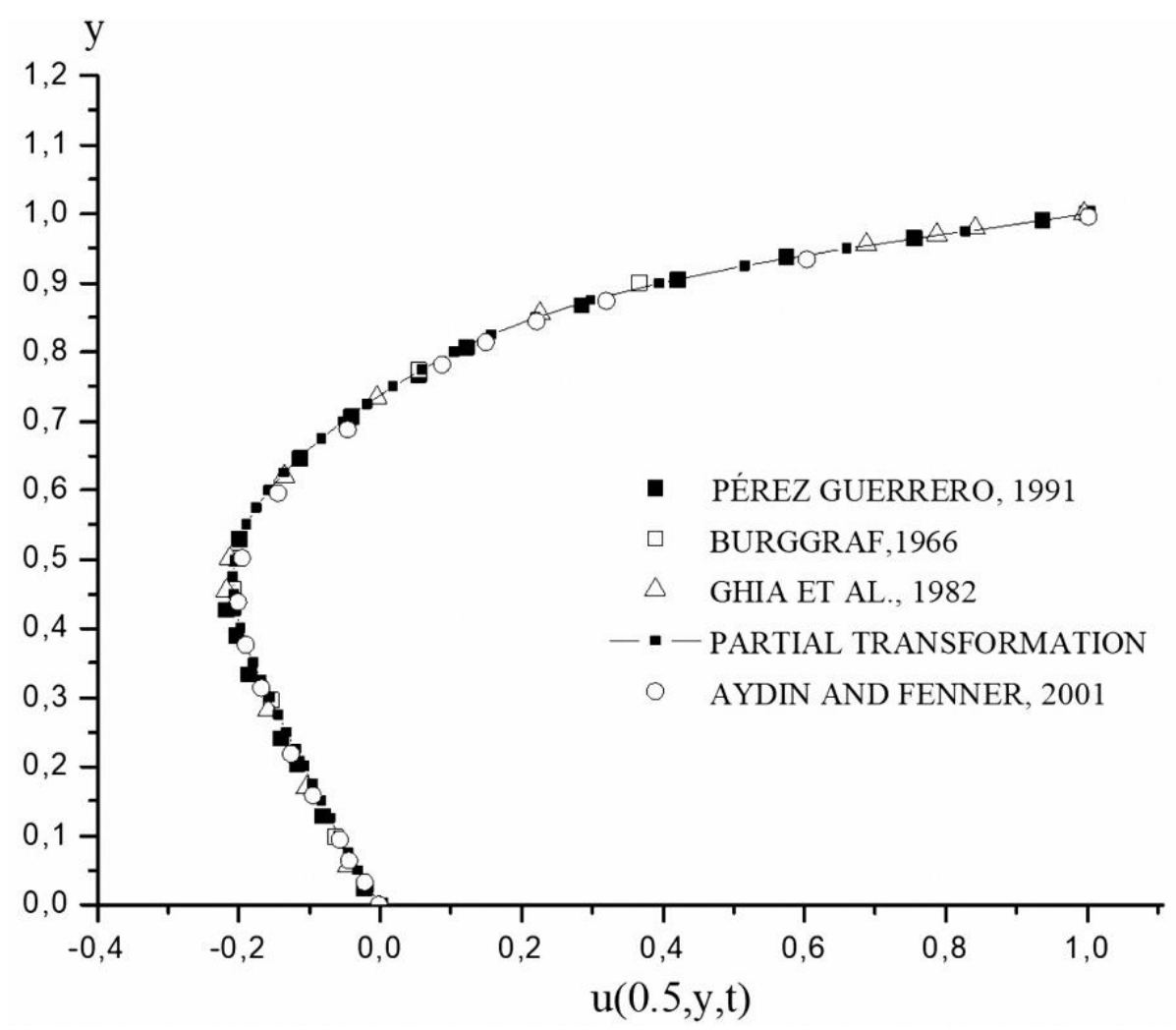


(a)

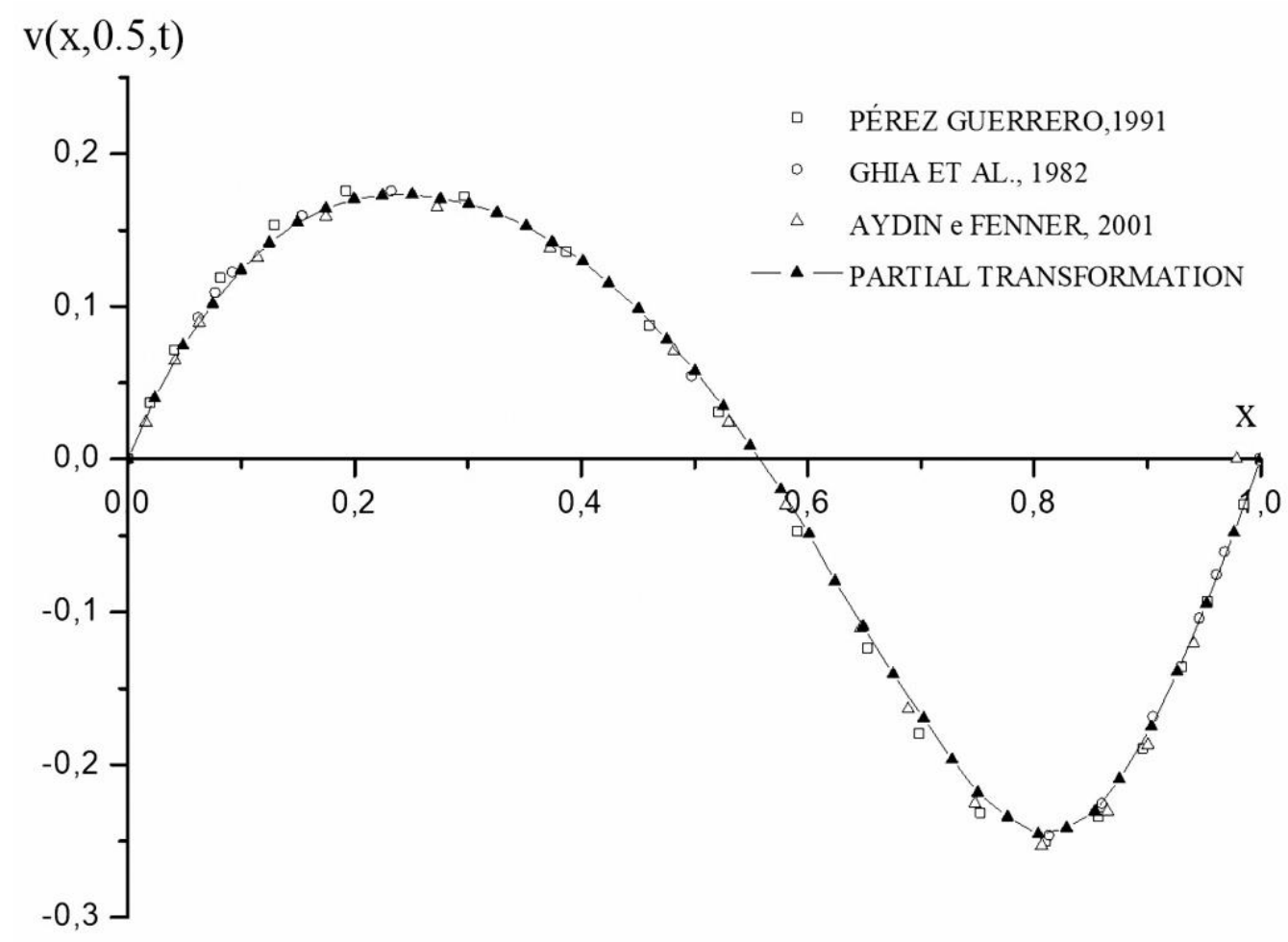

(b)

Figures 8: a) Horizontal and b) Vertical velocity components at the cavity centreline for $\mathrm{Re}=100$.

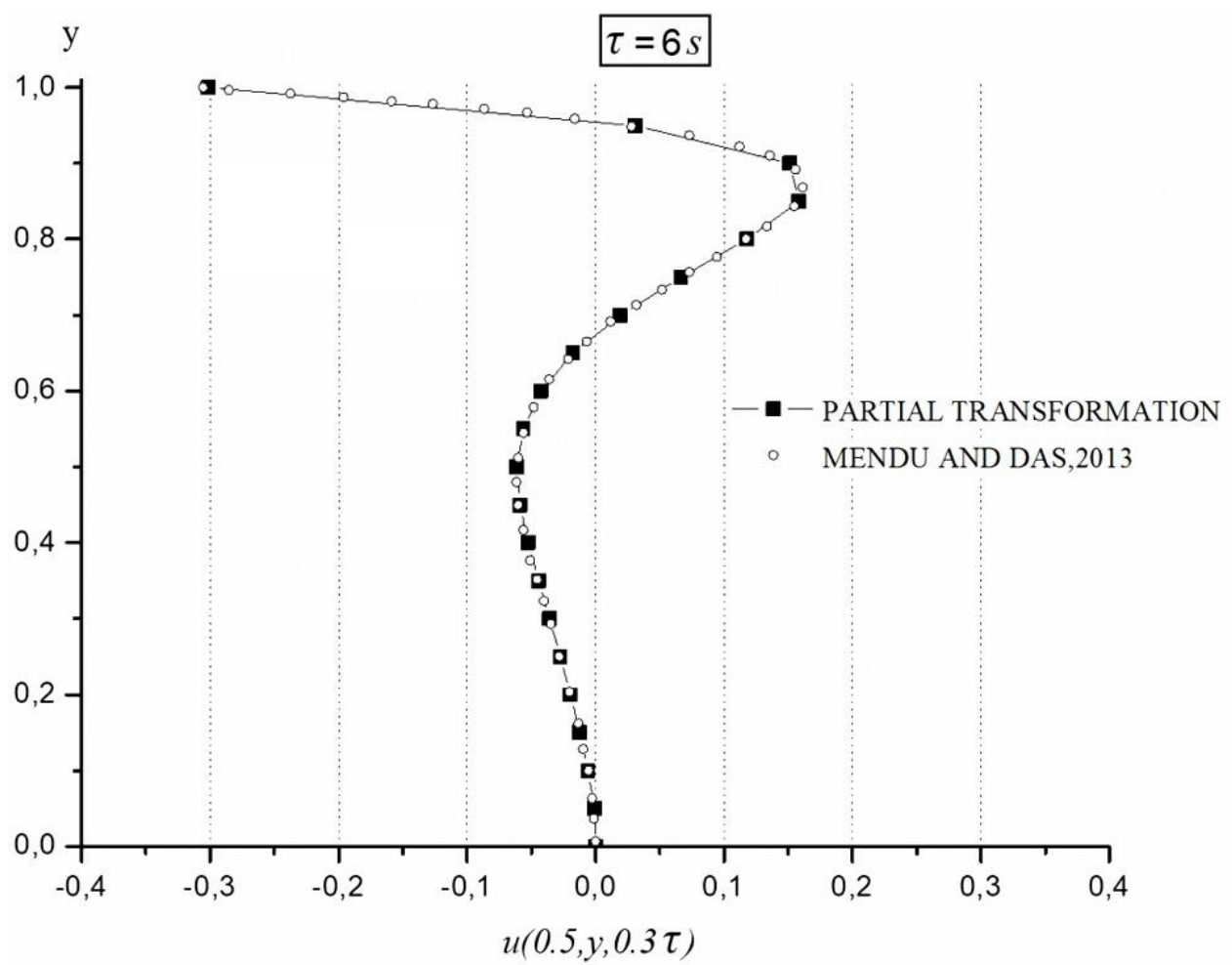


(a)

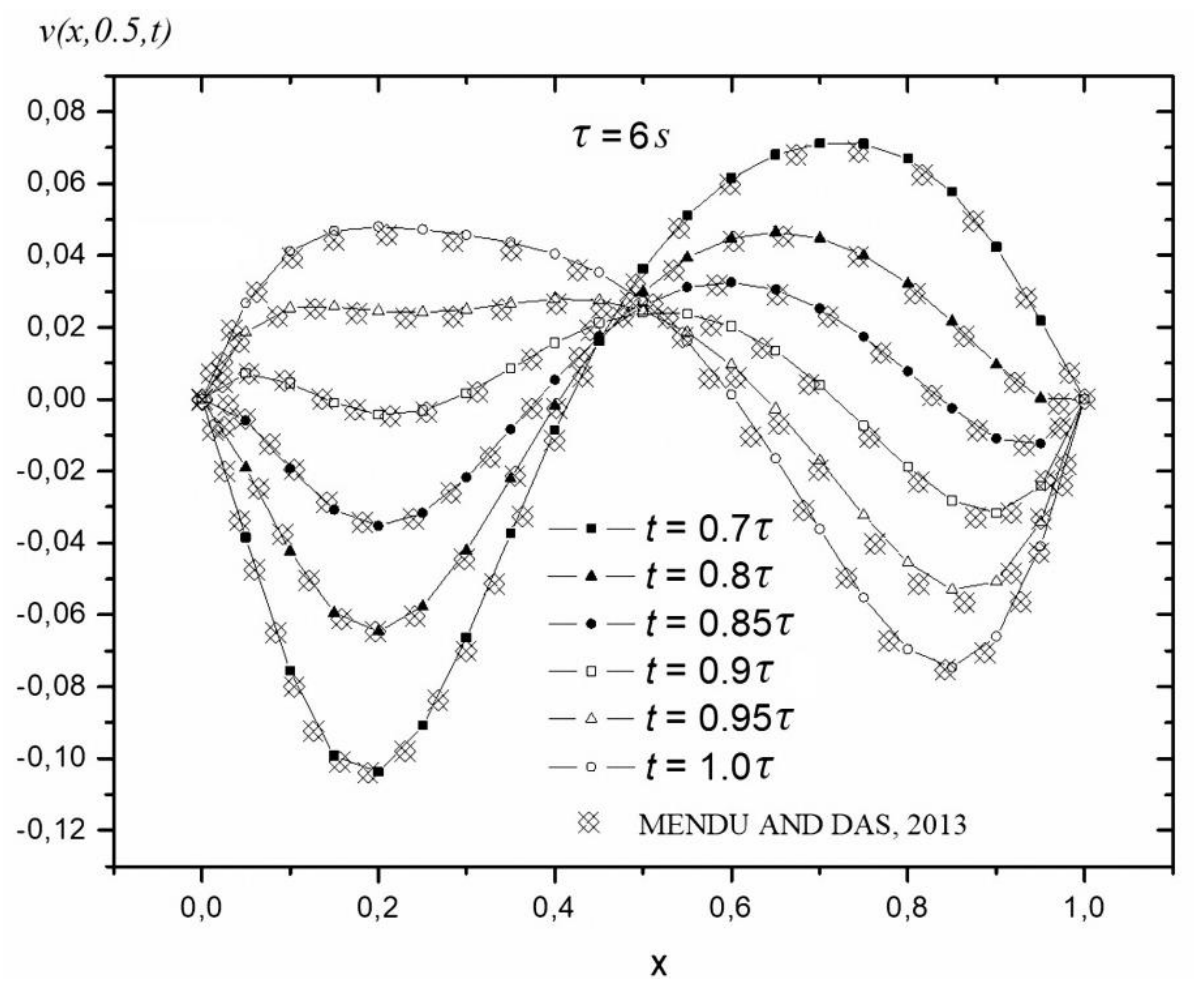

(b)

Figures 9: Comparison of the a)horizontal and b)vertical velocity components for the oscillating lid driven flow problem with $\mathrm{Re}=100$ and $\quad=2 / 6$.

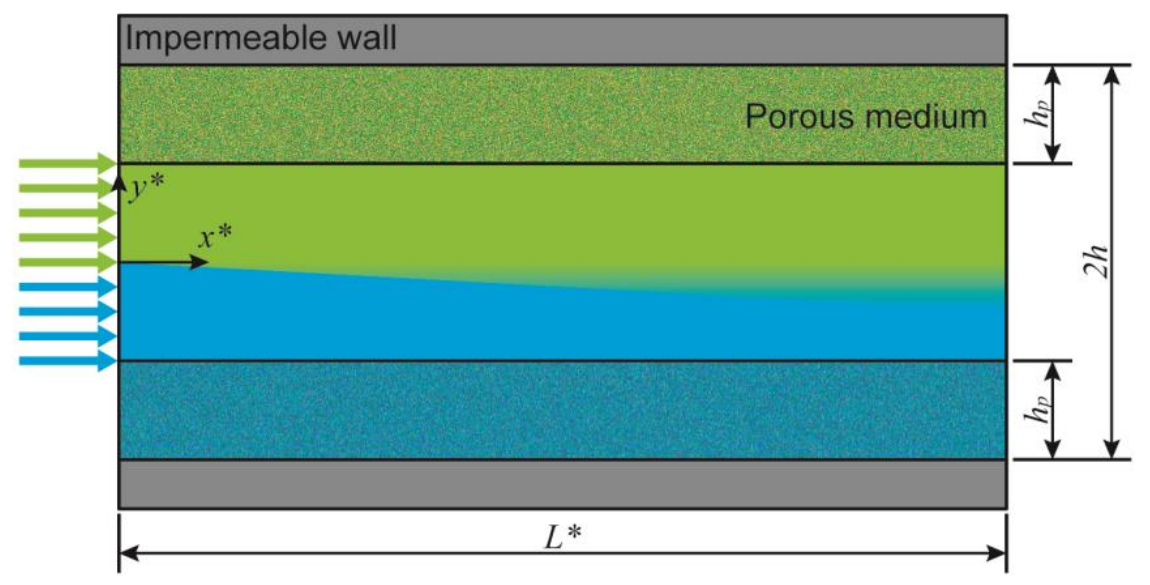

Figure 10: Illustration of the MRFB (Membraneless Redox Flow Battery) analyzed. Battery in flow-by configuration with porous carbon electrodes disposed symmetrically. 

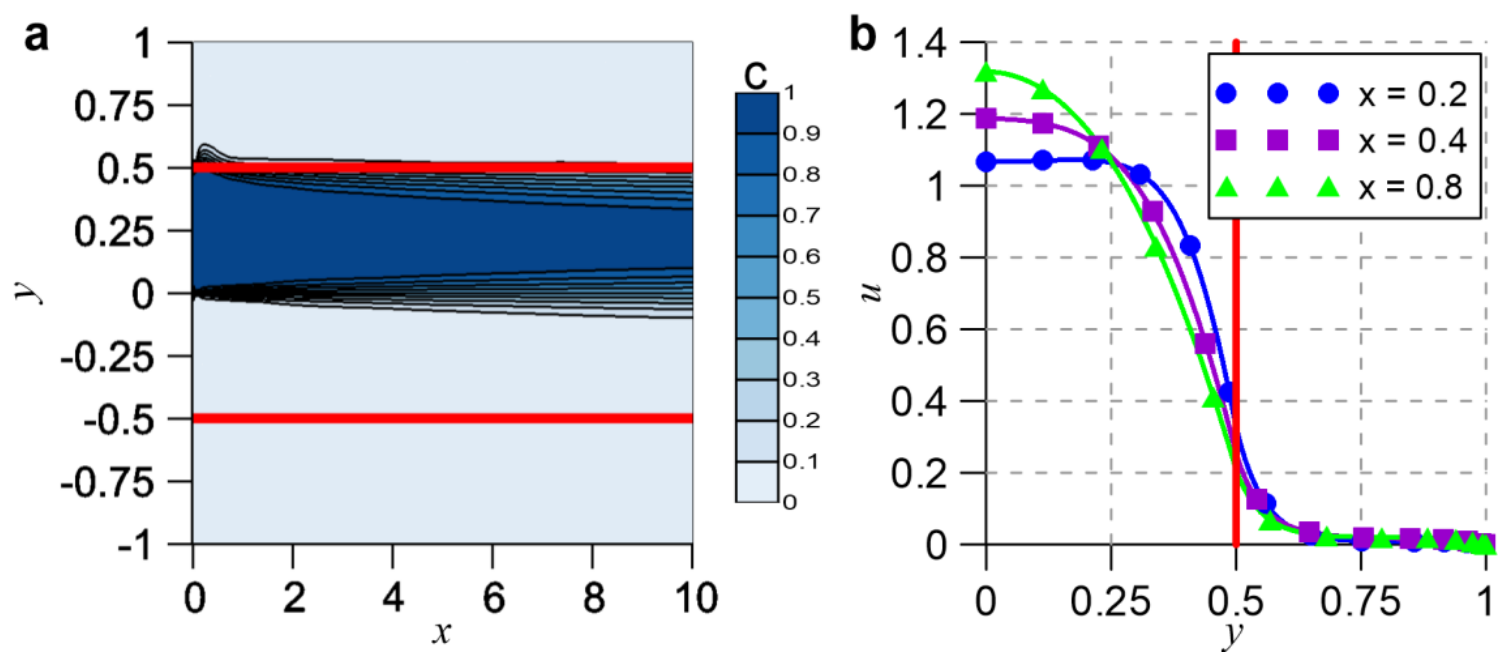

Figures 11: Concentration contours and horizontal component of the velocity vector varying with y for $\mathrm{Re}=50$ and $\mathrm{Da}=0.002$. a) Concentration contours. Horizontal red lines represent the inteface between fluid and porous media; b) Profiles of the horizontal velocity profile with a vertical red line representing the interface between fluid and porous medium. COMSOL results given as symbols and GITT results as solid lines. 